\title{
Analytic integration of real-virtual counterterms in NNLO jet cross sections II
}

\author{
Paolo Bolzoni, ${ }^{a}$ Sven-Olaf Moch, ${ }^{a}$ Gábor Somogyi ${ }^{b}$ and Zoltán Trócsányi ${ }^{c}$ \\ ${ }^{a} D E S Y$, \\ Platanenalle 6, D-15738 Zeuthen, Germany \\ ${ }^{b}$ Institute for Theoretical Physics, University of Zürich, \\ Winterthurerstrasse 190, CH-8057 Zürich, Switzerland \\ ${ }^{c}$ University of Debrecen and Institute of Nuclear Research of the Hungarian Academy of Sciences, \\ H-4001 Debrecen P.O.Box 51, Hungary \\ E-mail: paolo.bolzoni@desy.de, sven-olaf.moch@desy.de, \\ sgabi@physik.unizh.ch, z.trocsanyi@atomki.hu
}

ABSTRACT: We present analytic expressions of all integrals required to complete the explicit evaluation of the real-virtual integrated counterterms needed to define a recently proposed subtraction scheme for jet cross sections at next-to-next-to-leading order in QCD. We use the Mellin-Barnes representation of these integrals in $4-2 \epsilon$ dimensions to obtain the coefficients of their Laurent expansions around $\epsilon=0$. These coefficients are given by linear combinations of multidimensional Mellin-Barnes integrals. We compute the coefficients of such expansions in $\epsilon$ both numerically and analytically by complex integration over the Mellin-Barnes contours.

KEYWORDS: Jets, QCD

ARXIV EPRINT: 0905.4390 


\section{Contents}

1 Introduction $\quad 1$

2 Integrals needed for the integrated subtraction terms 3

2.1 Basic integrals 4

2.2 Nested integrals 5

$\begin{array}{lll}3 & \text { The method of Mellin-Barnes representations } & 7\end{array}$

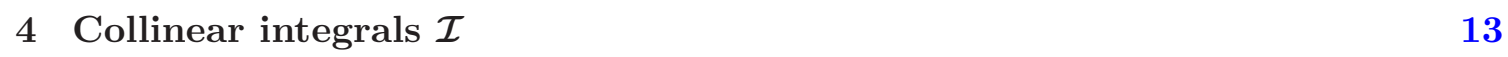

$5 \quad$ Nested collinear-type $\mathcal{I} * \mathcal{I}$ and $\mathcal{I} * \mathcal{J}$ integrals $\quad 15$

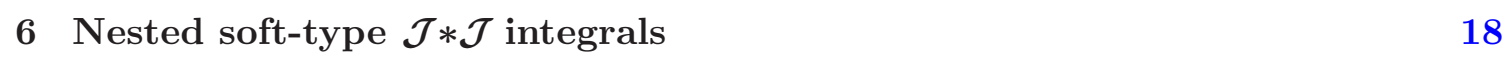

$\begin{array}{lll}7 & \text { Nested soft-collinear } \mathcal{K} * \mathcal{J} \text { integral } & 21\end{array}$

8 Conclusions $\quad 21$

\section{Introduction}

Precision predictions in perturbative Quantum Chromodynamics (QCD) at colliders demand calculating physical observables beyond leading order (LO) accuracy and, in the traditional approach to higher order predictions with fully differential kinematics, real and virtual corrections are separately evaluated. Integration over the phase space then requires a consistent treatment of the infrared singularities before any numerical computation may be performed. At next-to-leading order (NLO), infrared divergences can be handled using a subtraction scheme, which exploits the universal structure of the kinematical singularities of QCD matrix elements. The necessary (process-independent) counterterms regularize the virtual corrections at one loop and the real emission phase space integrals simultaneously [1].

At next-to-next-to-leading order (NNLO), the calculation of the radiative corrections to fully differential cross sections is a challenging problem and various extensions of the subtraction method at NNLO have been proposed, see e.g. refs. [2-5]. Currently, the available results for electron-positron annihilation at NNLO include total rates [6-8] and event shapes $[9,10]$ for the process $e^{+} e^{-} \rightarrow 3$ jets and are all based on the antenna subtraction method [11-13]. On the other hand for colorless final states, such as vector boson or Higgs boson production at hadron colliders dedicated subtraction schemes at NNLO $[14,15]$ have been applied. The infrared structure of scattering processes with three or more colored partons is involved if calculated at NNLO with the antenna subtraction method [16] - a fact 
which has motivated the formulation of alternative subtraction schemes. In particular, refs. [17-19] introduce a scheme for computing NNLO corrections to QCD jet cross sections for processes without colored partons in the initial state and an arbitrary number of massless particles (colored or colorless) in the final state. Very recently, following the steps of ref. [17], this subtraction scheme has been extended to cross sections for hadron-initiated processes [20], although yet to NLO accuracy only, but in a way which is NNLO-compatible.

Any subtraction scheme is of practical utility only after the counterterms for the regularization of the real emissions are integrated over the phase space of the unresolved partons. In the scheme of refs. [17-19] these counterterms are universal (but complete only for processes without colored particles in the initial state) and, therefore can be computed once and for all. Their knowledge is necessary to regularize the infrared divergences appearing in the virtual corrections. Some of the integrals needed explicitly in the so-called real-virtual counterterms of this scheme have been calculated in refs. [21, 22]. In the present paper we complete this task by computing all integrals needed for the the real-virtual counterterms in the subtraction scheme of refs. [17-19] by means of Mellin-Barnes (MB) representations. The use of MB integrals when dealing with Feynman integral calculus has proved powerful in the last years. MB integrals were first applied to Feynman integrals in refs. [23, 24] and pioneering work has been performed since then in refs. [25-27] (see also ref. [28] and references therein for many other examples). For a given integral the MB representation replaces the power of a sum in the integrand by a product of the individual terms of the sum raised to some other powers. This leads then to integrals over certain complex contours of $\Gamma$-functions. As a crucial point it is then very convenient with this MB representation to resolve all singularities in the limit $\epsilon=0$ within dimensional regularization, $d=4-2 \epsilon$. In this paper, we adapt the MB method to derive analytic expressions for all integrals appearing in the real-virtual counterterms of refs. [17-19].

Let us briefly discuss the merits of the analytic approach for the computation of the integrated subtraction terms. First of all, in a higher-order computation, the $\epsilon$ poles of the integrated subtraction terms need to cancel the corresponding $\epsilon$ poles coming from the loop matrix elements in the virtual corrections. The cancellation of these poles can be demonstrated most convincingly once the pole structure of the integrated subtraction terms is exhibited analytically. Second, in terms of speed and precision of the evaluation, analytic results are very fast and very accurate compared to numerical ones. Moreover, they demonstrate that the final result consists of smooth functions only. Nevertheless also the numerical evaluation of the integrated counterterms has its utility, because it serves as an independent check. Then, there are indeed some cases, where it is very difficult to find the analytic computation of the multi-dimensional MB integral and only the complex numerical integration can be carried out. In these cases, however, the method of MB integrals provides a fast and reliable way to obtain the final results with small numerical uncertainties. From a practical point of view, the combination of both, analytic and numerical evaluations of all MB integrals implies that the final results for the integrated real-virtual counterterms can be conveniently given e.g. in the form of interpolating tables which can be computed once and for all.

This suffices for any practical application, because in an actual computation the relative uncertainty associated with the numerical phase space integrations is generally much greater than that of the integrated subtraction terms. 


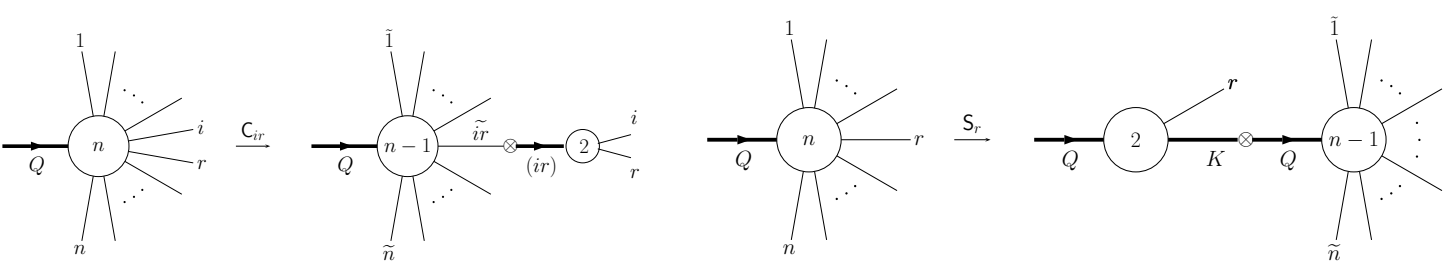

Figure 1. Graphical representations of the momentum mappings and the implied phase space factorization: collinear (left) and soft momentum mapping (right).

The outline of the paper is the following. In section 2 we briefly review the phase space integrals of the real-virtual corrections at NNLO and we define the integrals of the subtraction terms that we will consider in this paper. In section 3 we present a brief explanation of the method of MB representations. We outline the steps of our calculation and we also discuss explicitly an example to display the typical structure of the integrals we are interested in. In section 4 we complete the analytic evaluation of all integrals needed for integrated collinear counterterms. Next, in sections $5-7$ we compute also all different types of the nested integrals. Finally in section 8 we present the conclusions of this work.

\section{Integrals needed for the integrated subtraction terms}

The subtraction method developed in refs. $[18,19]$ relies on the universal soft and collinear factorization properties of QCD squared matrix elements. Once the subtraction scheme is defined, one has to integrate the subtraction terms over the factorized phase space of the unresolved parton(s). This is the content of the present work (see also ref. [21]).

There are two crucial elements in the formulation of a subtraction scheme beyond NLO. Firstly, the factorization formulae should disentangle the overlaps in soft-singular factors and collinear singularities in order to avoid multiple subtractions and a simple solution to this problem has been given in ref. [30]. Secondly, because the factorization formulae are valid only in the strict soft and collinear limits, they have to be extended to the whole phase space. Typically, this requires a mapping of the original $n$ momenta $\{p\}_{n}=\left\{p_{1}, \ldots, p_{n}\right\}$ in an $n$-parton matrix element at any order in perturbation theory to $m$ momenta $\{\tilde{p}\}_{m}=\left\{\tilde{p}_{1}, \ldots, \tilde{p}_{m}\right\}$ in such a way, that momentum conservation is preserved. Here $m$ denotes the number of hard partons and $n-m$ is the number of unresolved ones.

The original $n$-particle phase space of total momentum $Q$ reads

$$
\mathrm{d} \phi_{n}\left(p_{1}, \ldots, p_{n} ; Q\right)=\prod_{i=1}^{n} \frac{\mathrm{d}^{d} p_{i}}{(2 \pi)^{d-1}} \delta_{+}\left(p_{i}^{2}\right)(2 \pi)^{d} \delta^{(d)}\left(Q-\sum_{i=1}^{n} p_{i}\right)
$$

and, for a given mapping, one obtains the phase-space factorization as

$$
\mathrm{d} \phi_{n}\left(\{p\}_{n} ; Q\right)=\mathrm{d} \phi_{m}\left(\{\tilde{p}\}_{m} ; Q\right)\left[\mathrm{d} p_{n-m ; m}\left(\{p\}_{n-m} ; Q\right)\right],
$$

which was first introduced in ref. [1] in the context of computing QCD corrections at NLO. In this paper we are concerned with the integrals of the singly-unresolved counterterms 


\begin{tabular}{|c|c|c|}
\hline$\delta$ & Function & $g_{I}^{( \pm)}(z)$ \\
\hline 0 & $g_{A}$ & 1 \\
$\mp 1$ & $g_{B}^{( \pm)}$ & $(1-z)^{ \pm \epsilon}$ \\
0 & $g_{C}^{( \pm)}$ & $(1-z)^{ \pm \epsilon}{ }_{2} F_{1}( \pm \epsilon, \pm \epsilon, 1 \pm \epsilon, z)$ \\
\pm 1 & $g_{D}^{( \pm)}$ & ${ }_{2} F_{1}( \pm \epsilon, \pm \epsilon, 1 \pm \epsilon, 1-z)$ \\
\hline
\end{tabular}

Table 1. The values of $\delta$ and $g_{I}^{( \pm)}\left(z_{r}\right)$ for which eq. (2.5) needs to be evaluated.

(i.e. the case $m=1$ ), which imply two types of mappings:

$$
\begin{aligned}
& \{p\}_{n} \stackrel{\mathrm{C}_{i r}}{\longrightarrow}\{\tilde{p}\}_{n-1}^{(i r)}=\left\{\tilde{p}_{1}, \ldots, \tilde{p}_{i r}, \ldots, \tilde{p}_{n}\right\} \\
& \{p\}_{n} \stackrel{\mathrm{S}_{r}}{\longrightarrow}\{\tilde{p}\}_{n-1}^{(r)}=\left\{\tilde{p}_{1}, \ldots, \tilde{p}_{n}\right\} .
\end{aligned}
$$

In the collinear momentum mapping $\stackrel{\mathrm{C}_{i r}}{\longrightarrow}$ in eq. (2.3) the momenta $p_{i}^{\mu}$ and $p_{r}^{\mu}$ are replaced by a single momentum $\tilde{p}_{i r}^{\mu}$ and all other momenta are rescaled, while for soft-type subtractions, $\stackrel{\mathrm{S}_{r}}{\longrightarrow}$ in eq. (2.4) the momentum $p_{r}^{\mu}$, that may become soft, is missing from the set, and all other momenta are rescaled and transformed by a proper Lorentz transformation. Both momentum mappings and the corresponding factorization of the phase-space measure are represented graphically in figure 1 , where the symbol $\otimes$ stands for the convolution as implied by eq. (2.2). The integration of the singly-unresolved subtraction terms requires three basic types of integrals over the corresponding factorized phase space, as well as iterations of these (nested integrals are denoted by a $*$ ). All necessary integrals were derived in refs. [21, 22].

\subsection{Basic integrals}

The three basic integrals are those used in the collinear, soft and soft-collinear subtraction counterterms. The collinear integrals have the general form

$$
\begin{gathered}
\mathcal{I}\left(x ; \epsilon, \alpha_{0}, d_{0} ; \kappa, k, \delta, g_{I}^{( \pm)}\right)=x \int_{0}^{\alpha_{0}} \mathrm{~d} \alpha \alpha^{-1-(1+\kappa) \epsilon}(1-\alpha)^{2 d_{0}-1}[\alpha+(1-\alpha) x]^{-1-(1+\kappa) \epsilon} \\
\times \int_{0}^{1} \mathrm{~d} v[v(1-v)]^{-\epsilon}\left(\frac{\alpha+(1-\alpha) x v}{2 \alpha+(1-\alpha) x}\right)^{k+\delta \epsilon} g_{I}^{( \pm)}\left(\frac{\alpha+(1-\alpha) x v}{2 \alpha+(1-\alpha) x}\right)
\end{gathered}
$$

These integrals need to be known as a function of $x \in[0,1]$ in a Laurent-expansion in $\epsilon$ for $k=-1,0,1,2$. The necessary values of $\delta$ and the expressions for the functions $g_{I}^{( \pm)}$are given in table 1 . Here $\kappa=0,1$ for the first row and $\kappa=1$ for all other cases. Analytic expressions for all cases corresponding to the first two rows of table 1 were derived in ref. [21] and contain the first five terms in the $\epsilon$-expansion. We compute all cases anew and present our results explicitly in section 4 . The other parameters $\alpha_{0} \in(0,1]$ and $d_{0}$ in eq. (2.5) will be specified in section 3. Our analytic results for these integrals include all the coefficients of the poles in $\epsilon$ and the first three terms in the $\epsilon$-expansion. 
Next, the soft subtractions require the integral

$$
\begin{gathered}
\mathcal{J}\left(Y_{\tilde{i} \tilde{k}, Q} ; \epsilon, y_{0}, d_{0}^{\prime} ; \kappa\right)=-\left(4 Y_{\tilde{i} \tilde{k}, Q}\right)^{1+\kappa \epsilon} \frac{\Gamma^{2}(1-\epsilon)}{2 \pi \Gamma(1-2 \epsilon)} \Omega^{(1+\kappa \epsilon, 1+\kappa \epsilon)}(\cos \chi) \\
\times \int_{0}^{y_{0}} \mathrm{~d} y y^{-1-2(1+\kappa) \epsilon}(1-y)^{d_{0}^{\prime}+\kappa \epsilon}
\end{gathered}
$$

as a function of $Y_{\tilde{i} \tilde{k}, Q} \in[0,1]$ in a Laurent expansion around $\epsilon=0$, where $\Omega^{(i, k)}(\cos \chi)$ denotes the angular integral in $d$-dimensions

$$
\begin{aligned}
\Omega^{(i, k)}(\cos \chi)=\int_{-1}^{1} \mathrm{~d}(\cos \vartheta)(\sin \vartheta)^{-2 \epsilon} \int_{-1}^{1} \mathrm{~d}(\cos \varphi)(\sin \varphi)^{-1-2 \epsilon} \\
\quad \times(1-\cos \vartheta)^{-i}(1-\cos \chi \cos \vartheta-\sin \chi \sin \vartheta \cos \varphi)^{-k}
\end{aligned}
$$

with

$$
\cos \chi=1-2 Y_{\tilde{i} \tilde{k}, Q}
$$

For the present paper the exact definition of the kinematic variables $x$ and $Y_{\tilde{i} \tilde{k}, Q}$ is unimportant, nevertheless we recall their definition to make their physical meaning explicit. The kinematic variable $x$ is given by

$$
x=\frac{2 \tilde{p}_{i r} \cdot Q}{Q^{2}}
$$

where $\tilde{p}_{i r}$ is the momentum of the parent parton in the $(i r) \rightarrow i+r$ splitting, which appears on the right hand side of eq. (2.3) above, while $Q$ is the total incoming momentum. We note that in the strict collinear limit we have $\tilde{p}_{i r} \rightarrow p_{i}+p_{r}$. The kinematic variable $Y_{\tilde{i} \tilde{k}, Q}$ is defined as

$$
Y_{\tilde{i} \tilde{k}, Q}=\frac{1}{2} \frac{Q^{2}\left(\tilde{p}_{i} \cdot \tilde{p}_{k}\right)}{\left(\tilde{p}_{i} \cdot Q\right)\left(\tilde{p}_{k} \cdot Q\right)}
$$

Finally, the soft-collinear subtractions lead to the integral

$$
\begin{aligned}
\mathcal{K}\left(\epsilon, y_{0}, d_{0}^{\prime} ; \kappa\right)=2 & \int_{0}^{y_{0}} \mathrm{~d} y y^{-(2+\kappa) \epsilon}(1-y)^{d_{0}^{\prime}-1} \int_{-1}^{1} \mathrm{~d}(\cos \vartheta)(\sin \vartheta)^{-2 \epsilon} \\
& \times\left[1+\frac{2(1-y)}{y(1-\cos \vartheta)}\right]^{1+\kappa \epsilon} \frac{\Gamma^{2}(1-\epsilon)}{2 \pi \Gamma(1-2 \epsilon)} \int_{-1}^{1} \mathrm{~d}(\cos \varphi)(\sin \varphi)^{-1-2 \epsilon},
\end{aligned}
$$

which does not depend on kinematical variables. The integrals $\mathcal{J}$ and $\mathcal{K}$ in eqs. (2.6) and (2.11) have been computed in ref. [21] for all relevant parameters in $y_{0}, d_{0}^{\prime}$ and $\kappa$. We have evaluated these soft and soft-collinear integrals, too, and we have checked that the two results agree numerically. We do not deal with the cases $\mathcal{J}$ and $\mathcal{K}$ in this paper.

\section{$2.2 \quad$ Nested integrals}

In a NNLO computation, also iterations of the above integrals appear. In this paper we complete the list of nested integrals necessary for the integrated real-virtual counterterms, in particular we cover all cases which have not been addressed in ref. [21]. 
Of the nested integrals, which we generally denote by a star $*$, three are collinear integrals with one of the basic types in its argument,

$$
\begin{aligned}
& \mathcal{I}_{*} \mathcal{I}_{i}(x ;\left.\epsilon, \alpha_{0}, d_{0} ; k, l\right)=x \int_{0}^{\alpha_{0}} \mathrm{~d} \alpha \int_{0}^{1} \mathrm{~d} v \alpha^{-1-\epsilon}(1-\alpha)^{2 d_{0}-1}[\alpha+(1-\alpha) x]^{-1-\epsilon} \\
& \times[v(1-v)]^{-\epsilon}\left[\frac{\alpha+(1-\alpha) x v}{2 \alpha+(1-\alpha) x}\right]^{k} \mathcal{I}\left(x \frac{\alpha+(1-\alpha) x(1-v)}{2 \alpha+(1-\alpha) x} ; \epsilon, \alpha_{0}, d_{0} ; 0, l, 0,1\right), \\
& \mathcal{I}_{*} \mathcal{I}_{r}\left(x ; \epsilon, \alpha_{0}, d_{0} ; k, l\right)=x \int_{0}^{\alpha_{0}} \mathrm{~d} \alpha \int_{0}^{1} \mathrm{~d} v \alpha^{-1-\epsilon}(1-\alpha)^{2 d_{0}-1}[\alpha+(1-\alpha) x]^{-1-\epsilon} \\
& \times[v(1-v)]^{-\epsilon}\left[\frac{\alpha+(1-\alpha) x v}{2 \alpha+(1-\alpha) x}\right]^{k} \mathcal{I}\left(x \frac{\alpha+(1-\alpha) x v}{2 \alpha+(1-\alpha) x} ; \epsilon, \alpha_{0}, d_{0} ; 0, l, 0,1\right),
\end{aligned}
$$

which we need for $k, l=-1,0,1,2$, and

$$
\begin{aligned}
\mathcal{I} * \mathcal{J}\left(x ; \epsilon, \alpha_{0}, d_{0}, y_{0}, d_{0}^{\prime} ; k\right)=x & \int_{0}^{\alpha_{0}} \mathrm{~d} \alpha \int_{0}^{1} \mathrm{~d} v \alpha^{-1-\epsilon}(1-\alpha)^{2 d_{0}-1}[\alpha+(1-\alpha) x]^{-1-\epsilon} \\
& \times[v(1-v)]^{-\epsilon}\left[\frac{\alpha+(1-\alpha) x v}{2 \alpha+(1-\alpha) x}\right]^{k} \\
& \times \mathcal{J}\left(\frac{\alpha(\alpha+(1-\alpha) x)(2 \alpha+(1-\alpha) x)^{2}}{(\alpha+(1-\alpha) x v)(\alpha+(1-\alpha) x(1-v)) x^{2}} ; \epsilon, y_{0}, d_{0}^{\prime}, 0\right),
\end{aligned}
$$

for $k=-1,0,1,2$. Both, $\mathcal{I}_{*} \mathcal{I}$ and $\mathcal{I}_{*} \mathcal{J}$ are needed as a function of $x \in[0,1]$ in an $\epsilon$-expansion with $\mathcal{I}$ and $\mathcal{J}$ given in eqs. (2.5) and (2.6), respectively. A discussion about the choice of the relevant parameters $\alpha_{0}, d_{0}, y_{0}$ and $d_{0}^{\prime}$ is given at the end of section 3 and details of the computation are also given in section 5 .

Three other iterated integrals are defined as soft integrals with other soft integrals appearing in the argument,

$$
\begin{aligned}
& \mathcal{J} * \mathcal{J}_{i k}\left(Y_{\tilde{i} \tilde{k}, Q} ; \epsilon, y_{0}, d_{0}^{\prime}\right)=-8 Y_{\tilde{i} \tilde{k}, Q} \frac{\Gamma^{2}(1-\epsilon)}{2 \pi \Gamma(1-2 \epsilon)} \int_{-1}^{1} \mathrm{~d}(\cos \vartheta)(\sin \vartheta)^{-2 \epsilon} \\
& \times \int_{-1}^{1} \mathrm{~d}(\cos \varphi)(\sin \varphi)^{-1-2 \epsilon}(1-\cos \vartheta)^{-1} \\
& \times \int_{0}^{y_{0}} \mathrm{~d} y y^{-1-2 \epsilon}(1-y)^{d_{0}^{\prime}}[2-(1+\cos \chi) \cos \vartheta-\sin \chi \sin \vartheta \cos \varphi]^{-1} \\
& \times \mathcal{J}\left(\frac{4(1-y) Y_{\tilde{i} \tilde{k}, Q}}{[2-y(1+\cos \vartheta)][2-y(1+\cos \chi \cos \vartheta+\sin \chi \sin \vartheta \cos \varphi)]} ; \epsilon, y_{0}, d_{0}^{\prime}, 0\right), \\
& \mathcal{J} * \mathcal{J}_{i r}\left(Y_{\tilde{i} \tilde{k}, Q} ; \epsilon, y_{0}, d_{0}^{\prime}\right)=-8 Y_{\tilde{i} \tilde{k}, Q} \frac{\Gamma^{2}(1-\epsilon)}{2 \pi \Gamma(1-2 \epsilon)} \int_{-1}^{1} \mathrm{~d}(\cos \vartheta)(\sin \vartheta)^{-2 \epsilon} \\
& \times \int_{-1}^{1} \mathrm{~d}(\cos \varphi)(\sin \varphi)^{-1-2 \epsilon}(1-\cos \vartheta)^{-1} \\
& \times[2-(1+\cos \chi) \cos \vartheta-\sin \chi \sin \vartheta \cos \varphi]^{-1} \\
& \times \int_{0}^{y_{0}} \mathrm{~d} y y^{-1-2 \epsilon}(1-y)^{d_{0}^{\prime}} \mathcal{J}\left(\frac{(1-\cos \vartheta)}{2-y(1+\cos \vartheta)} ; \epsilon, y_{0}, d_{0}^{\prime}, 0\right),
\end{aligned}
$$


and

$$
\begin{aligned}
\mathcal{J} * \mathcal{J}_{k r}\left(Y_{\tilde{i} \tilde{k}, Q} ; \epsilon, y_{0}, d_{0}^{\prime}\right)=- & 8 Y_{\tilde{i} \tilde{k}, Q} \frac{\Gamma^{2}(1-\epsilon)}{2 \pi \Gamma(1-2 \epsilon)} \int_{-1}^{1} \mathrm{~d}(\cos \vartheta)(\sin \vartheta)^{-2 \epsilon} \\
& \times \int_{-1}^{1} \mathrm{~d}(\cos \varphi)(\sin \varphi)^{-1-2 \epsilon}(1-\cos \vartheta)^{-1} \\
& \times \int_{0}^{y_{0}} \mathrm{~d} y y^{-1-2 \epsilon}(1-y)^{d_{0}^{\prime}}[2-(1+\cos \chi) \cos \vartheta-\sin \chi \sin \vartheta \cos \varphi]^{-1} \\
& \times \mathcal{J}\left(\frac{(1-\cos \chi \cos \vartheta-\sin \chi \sin \vartheta \cos \varphi)}{2-y(1+\cos \chi \cos \vartheta+\sin \chi \sin \vartheta \cos \phi)} ; \epsilon, y_{0}, d_{0}^{\prime}, 0\right),
\end{aligned}
$$

with $\mathcal{J}$ given in eq. (2.6). The three integrals in eqs. (2.15)-(2.17) need to be calculated for $Y_{\tilde{i} \tilde{k}, Q} \in[0,1]$ as expansion in $\epsilon$. Explicit results and details of the computation (and values for the parameters $y_{0}$ and $d_{0}^{\prime}$ ) for these integrals are presented in sections 3 and 6 .

The final case is when the soft integral appears in the argument of a soft-collinear one,

$$
\begin{aligned}
\mathcal{K} * \mathcal{J}\left(\epsilon, y_{0}, d_{0}^{\prime}\right)= & 2 \frac{\Gamma^{2}(1-\epsilon)}{2 \pi \Gamma(1-2 \epsilon)} \int_{-1}^{1} \mathrm{~d}(\cos \vartheta)(\sin \vartheta)^{-2 \epsilon} \\
& \times \int_{-1}^{1} \mathrm{~d}(\cos \varphi)(\sin \varphi)^{-1-2 \epsilon} \int_{0}^{y_{0}} \mathrm{~d} y y^{-1-2 \epsilon}(1-y)^{d_{0}^{\prime}-1} \\
& \quad \times \frac{2-y(1+\cos \vartheta)}{1-\cos \vartheta} \mathcal{J}\left(\frac{1-\cos \vartheta}{2-y(1+\cos \vartheta)} ; \epsilon, y_{0}, d_{0}^{\prime}, 0\right),
\end{aligned}
$$

which is again independent of the kinematics, i.e. the coefficients of the expansion in $\epsilon$ are pure numbers. Details of the computation and the parameters $y_{0}$ and $d_{0}^{\prime}$ are given in sections 3 and 7 .

\section{The method of Mellin-Barnes representations}

In this section we briefly review the essential steps in the derivation of $\mathrm{MB}$ representations for the integrals of sections 2.1 and 2.2. The starting point is the well known basic formula,

$$
\frac{1}{(a+b)^{\nu}}=\frac{1}{\Gamma(\nu)} \int_{q-\mathrm{i} \infty}^{q+\mathrm{i} \infty} \frac{\mathrm{d} z}{2 \pi i} a^{-\nu-z} b^{z} \Gamma(\nu+z) \Gamma(-z),
$$

where $\nu$ and $q$ are real numbers (the case of $\nu=0$ is trivial) and $q$ sets the asymptotic position of the integration contour. The application of eq. (3.1) to Feynman integral calculus was initiated in refs. [23, 24] (see also ref. [28]) and is an algorithmic procedure which can be completely automatized, as e.g. in the Ambre.m package [31] in MATHEMATICA.

In general, the contour in eq. (3.1) is not necessarily a straight line and its standard definition is such that the poles of $\Gamma(\nu+z)$ (at $z=-i-\nu$ with $i$ being non-negative integer) are all to the left and the poles of $\Gamma(-z)$ (at non-negative integers) are all to the right of it. The condition on the poles of the $\Gamma$-functions can be satisfied by such a contour in eq. (3.1) if and only if $q<0$ and $\nu>0$. However, as a key observation, ref. [27] realized straight-line contours parallel to the imaginary axis in an algorithmic way. If $\nu<0$, we start with a curved contour that fulfills the condition on the pole and then deform it into a straight 

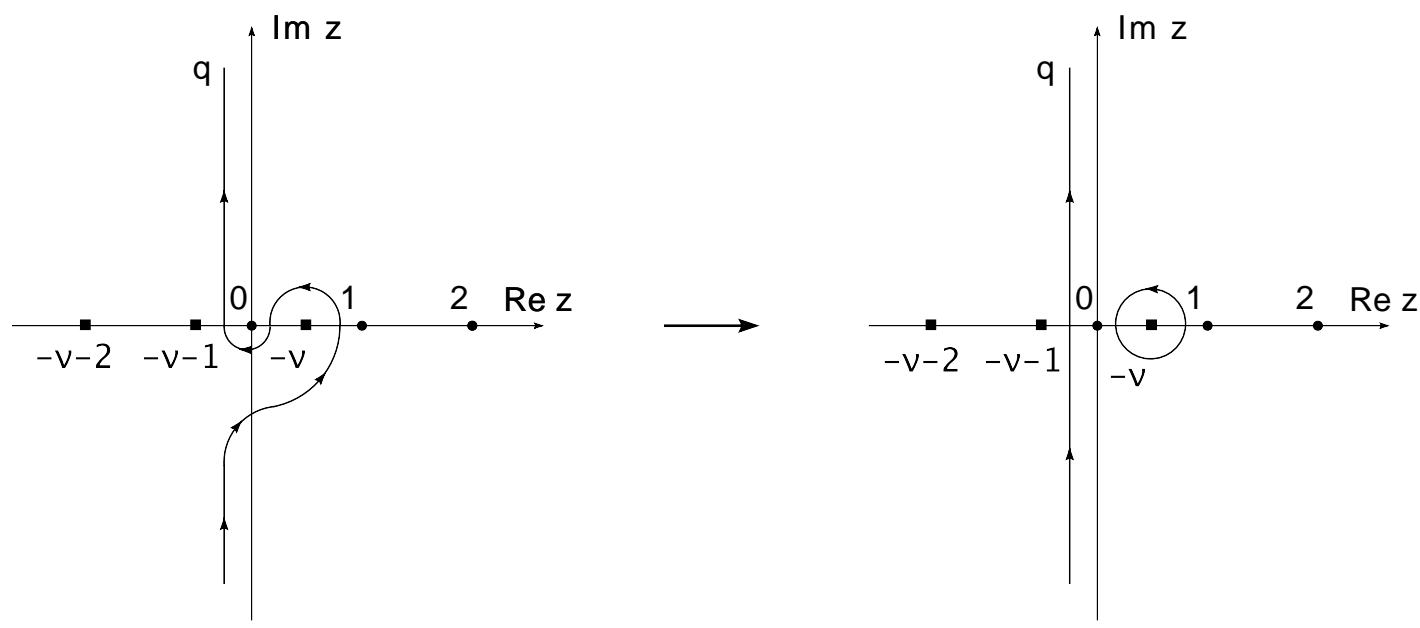

Figure 2. The deformation of a curved contour into the sum of a straight line and a circle for $\nu=-1 / 2$ and $q=-1 / 4$.

line taking into account the residua of the crossed poles according to Cauchy's theorem. This procedure lends itself to implementation in computer codes for the evaluation and manipulation of MB integrals, such as in the MB.m package [29].

For instance in eq. (3.1), if $\nu=-1 / 2$, a possible good choice for $q$ is $q=-1 / 4$. The curved contour depicted in figure 2 on the left fulfills the conditions on the poles of the $\Gamma$-functions. Contour deformation results in a straight line and a circle around the pole at $z=-\nu=1 / 2$ as shown graphically in figure 2 on the right. Therefore, the MB representation of $(a+b)^{1 / 2}$ in terms of a vertical straight line contour is given by

$$
(a+b)^{1 / 2}=\frac{1}{\Gamma(-1 / 2)} \int_{-1 / 4-\mathrm{i} \infty}^{-1 / 4+\mathrm{i} \infty} \frac{\mathrm{d} z}{2 \pi i} a^{1 / 2-z} b^{z} \Gamma(-1 / 2+z) \Gamma(-z)+\sqrt{b},
$$

where the first term corresponds to the integral along the straight line with $q=-1 / 4$ and the second one to the integral along a circle surrounding the pole in $z=1 / 2$ and evaluated according to Cauchy's theorem. Note that eq. (3.1) is not valid for negative integer values of $\nu$ because of $\Gamma(\nu)$ in the denominator. In these cases we use the binomial expansion

$$
\frac{1}{(a+b)^{\nu}}=\sum_{i=0}^{-\nu}\left(\begin{array}{c}
-\nu \\
i
\end{array}\right) a^{i} b^{-\nu-i}, \quad \text { for } \nu \text { being negative integer }
$$

We use eqs. (3.1) and (3.3) to convert all sums in the integrands of the soft, collinear and iterated integrals of section 2 into products. Then, we apply the relations

$$
(1-x)=\int_{0}^{1} \mathrm{~d} y y \delta(1-x-y)
$$

and

$$
\int_{0}^{1} \prod_{i=1}^{n} \mathrm{~d} x_{i} x_{i}^{p_{i}-1} \delta\left(1-\sum_{j=1}^{n} x_{j}\right)=\prod_{i=1}^{n} \Gamma\left(p_{i}\right) / \Gamma\left(\sum_{j=1}^{n} p_{j}\right),
$$


to obtain a representation of the original integrals in terms of MB integrals where all the integrations over $\alpha, v, \cos (\theta), \cos (\phi)$ are performed and only complex integrations along straight lines parallel to the imaginary axis are left following the procedure discussed below eq. (3.1). Upon deformation of the curved complex contours all singularities in $\epsilon$ are extracted so that it is safe to expand in $\epsilon$ around zero before doing the complex integration. In this way, the MB representations of the required coefficients of the Laurent expansions of the integrals of section 2 are obtained. In the next step we convert the complex contour integrations into harmonic sums using Cauchy's theorem and finally we evaluate the sums. For the computation of all the harmonic sums we have used algorithms for harmonic and nested sums of refs. [32, 33] as implemented in the XSummer package [34]. Typically, symbolic summation of single-scale nested sums leads at intermediate stages of the calculation to harmonic polylogarithms (HPLs) (see ref. [35] for a definition). However, in all cases where analytic result have been obtained by summing series of residues, the HPLs could be converted to standard polylogarithms (see also ref. [21] for a discussion of the class of functions appearing in the integrated real-virtual counterterms).

As an example let us consider the following integral:

$$
\mathcal{E}\left(x ; \epsilon, d_{0}\right)=x^{2} \int_{0}^{1} \mathrm{~d} \alpha \alpha^{-1-\epsilon}(1-\alpha)^{2 d_{0}}[\alpha+(1-\alpha) x]^{-1-\epsilon}[2 \alpha+(1-\alpha) x]^{-1},
$$

which is a typical contribution to the collinear integrals defined in eq. (2.5). The integral in eq. (3.6) is clearly divergent in the limit $\epsilon=0$ due to the factor $\alpha^{-1-\epsilon}$ in its integrand. The first step is to write the MB representation of the integral in eq. (3.6). To do this we use eq. (3.1) twice then eqs. (3.4) and (3.5). We obtain

$$
\begin{aligned}
\mathcal{E}\left(x ; \epsilon, d_{0}\right)= & \int_{q_{1}-\mathrm{i} \infty}^{q_{1}+\mathrm{i} \infty} \frac{\mathrm{d} z_{1}}{2 \pi i} \int_{q_{2}-\mathrm{i} \infty}^{q_{2}+\mathrm{i} \infty} \frac{\mathrm{d} z_{2}}{2 \pi i} 2^{z_{2}} x^{-\epsilon-z_{1}-z_{2}} \\
& \times \Gamma\left(\begin{array}{c}
-z_{1},-z_{2}, 2 d_{0}-1-\epsilon-z_{1}-z_{2}, 1+\epsilon+z_{1}, 1+z_{2},-\epsilon+z_{1}+z_{2} \\
2 d_{0}-1-2 \epsilon, 1+\epsilon
\end{array}\right),
\end{aligned}
$$

where we have introduced the notation

$$
\Gamma\left(\begin{array}{l}
a_{1}, a_{2}, \ldots, a_{n} \\
b_{1}, b_{2}, \ldots, b_{m}
\end{array}\right)=\prod_{i=1}^{n} \Gamma\left(a_{i}\right) / \prod_{j=1}^{m} \Gamma\left(b_{j}\right) .
$$

For $d_{0} \geq 2$ we choose $q_{1}=-1 / 4$ and $q_{2}=-1 / 8$ and curved contours such that the real parts of the arguments of all $\Gamma$-functions remain positive on them. Note that this implements the requirement that the contour separates the left poles from the right ones. In order to use straight-line contours, we must add contributions from two residua: the first is due to the residue coming from the pole in $z_{2}=\epsilon-z_{1}$ and then in the resulting one-dimensional MB integral the second is due to the residue in $z_{1}=\epsilon$. Adding these contributions to the starting representation of eq. (3.7), we find the MB representation of eq. (3.6) with $\epsilon$ close 
to zero to be given by:

$$
\begin{aligned}
\mathcal{E}\left(x ; \epsilon, d_{0}\right)= & x^{-2 \epsilon} \Gamma\left(\begin{array}{c}
-\epsilon, 1+2 \epsilon \\
1+\epsilon
\end{array}\right) \\
& +\int_{q_{1}-\mathrm{i} \infty}^{q_{1}+\mathrm{i} \infty} \frac{\mathrm{d} z_{1}}{2 \pi i} 2^{\epsilon-z_{1}} x^{-2 \epsilon} \Gamma\left(\begin{array}{c}
-z_{1},-\epsilon+z_{1}, 1+\epsilon+z_{1}, 1+\epsilon-z_{1} \\
1+\epsilon
\end{array}\right) \\
& +\int_{q_{1}-\mathrm{i} \infty}^{q_{1}+\mathrm{i} \infty} \frac{\mathrm{d} z_{1}}{2 \pi i} \int_{q_{2}-\mathrm{i} \infty}^{q_{2}+\mathrm{i} \infty} \frac{\mathrm{d} z_{2}}{2 \pi i} 2^{z_{2}} x^{-\epsilon-z_{1}-z_{2}} \\
& \times \Gamma\left(\begin{array}{c}
-z_{1},-z_{2}, 2 d_{0}-1-\epsilon-z_{1}-z_{2}, 1+\epsilon+z_{1}, 1+z_{2},-\epsilon+z_{1}+z_{2} \\
2 d_{0}-1-2 \epsilon, 1+\epsilon
\end{array}\right) .
\end{aligned}
$$

At this point we see that the singularity in $\epsilon=0$ is isolated in the first term of this equation. In particular the pole comes from the factor $\Gamma(-\epsilon)$ of this term. This shows that the extraction of poles comes out in a very convenient way: in practice we have only deformed contours and computed residua. As a matter of fact, this is one of the strong points in the application of MB methods to phase space integrals, and the straightforward way of extracting infrared poles has been already discussed in refs. [36, 37].

As a next step we can perform the expansion around $\epsilon=0$ and we obtain

$$
\begin{aligned}
\mathcal{E}\left(x ; \epsilon, d_{0}\right)=- & \frac{1}{\epsilon}+2 \log (x)+\int_{q_{1}-\mathrm{i} \infty}^{q_{1}+\mathrm{i} \infty} \frac{\mathrm{d} z_{1}}{2 \pi i} 2^{-z_{1}} \Gamma\left(1-z_{1},-z_{1}, z_{1}, 1+z_{1}\right) \\
& +\int_{q_{1}-\mathrm{i} \infty}^{q_{1}+\mathrm{i} \infty} \frac{\mathrm{d} z_{1}}{2 \pi i} \int_{q_{2}-\mathrm{i} \infty}^{q_{2}+\mathrm{i} \infty} \frac{\mathrm{d} z_{2}}{2 \pi i} 2^{z_{2}} x^{-z_{1}-z_{2}} \\
& \times \Gamma\left(\begin{array}{c}
-z_{1},-z_{2}, 2 d_{0}-1-z_{1}-z_{2}, 1+z_{1}, 1+z_{2}, z_{1}+z_{2} \\
2 d_{0}-1
\end{array}\right) .
\end{aligned}
$$

The first integral can be easily computed. We close the contour to the right and compute the residua coming from the poles enclosed in it at $z_{1}=n ; n \geq 0$. The residua are given by $(1 / 2)^{n} \log (2)$. Thus, multiplying by an overall minus sign due to the clockwise orientation of the contour, we find

$$
\int_{q_{1}-\mathrm{i} \infty}^{q_{1}+\mathrm{i} \infty} \frac{\mathrm{d} z_{1}}{2 \pi i} 2^{-z_{1}} \Gamma\left(1-z_{1},-z_{1}, z_{1}, 1+z_{1}\right)=-\sum_{n=0}^{\infty}(1 / 2)^{n} \log (2)=-2 \log (2) .
$$

Next we evaluate the second integral closing both contours to the left. We begin with the integration over the variable $z_{1}$ and we have two different $\Gamma$-functions that contribute with poles. The first one is $\Gamma\left(1+z_{1}\right)$ which exhibits poles in $z_{1}=-n ; n \geq 1$ and the second one is $\Gamma\left(z_{1}+z_{2}\right)$ which contributes with poles in $z_{1}=-n-z_{2} ; n \geq 1$. Computing these 
residua, we obtain for the second integral

$$
\begin{gathered}
\int_{q_{1}-\mathrm{i} \infty}^{q_{1}+\mathrm{i} \infty} \frac{\mathrm{d} z_{1}}{2 \pi i} \int_{q_{2}-\mathrm{i} \infty}^{q_{2}+\mathrm{i} \infty} \frac{\mathrm{d} z_{2}}{2 \pi i} 2^{z_{2}} x^{-z_{1}-z_{2}} \Gamma\left(\begin{array}{c}
-z_{1},-z_{2}, 2 d_{0}-1-z_{1}-z_{2}, 1+z_{1}, 1+z_{2}, z_{1}+z_{2} \\
2 d_{0}-1
\end{array}\right) \\
=\sum_{n=1}^{\infty} \int_{q_{2}-\mathrm{i} \infty}^{q_{2}+\mathrm{i} \infty} \frac{\mathrm{d} z_{2}}{2 \pi i}\left[\frac{(-1)^{n+1}}{(n-1) !} 2^{z_{2}} x^{n-z_{2}} \Gamma\left(\begin{array}{c}
n,-z_{2}, 2 d_{0}-1+n-z_{2}, 1+z_{2},-n+z_{2} \\
2 d_{0}-1
\end{array}\right)\right] . \\
\left.+\frac{(-x)^{n}}{n !} 2^{z_{2}} \Gamma\left(\begin{array}{c}
2 d_{0}-1+n, 1-n-z_{2},-z_{2}, 1+z_{2}, n+z_{2} \\
2 d_{0}-1
\end{array}\right)\right]
\end{gathered}
$$

Now we can do the remaining integration over $z_{2}$. In this case the poles of both the integrands are in $z_{2}=-m ; m \geq 1$ and the corresponding residua are given by

$$
\begin{aligned}
\mathcal{E}\left(x ; \epsilon, d_{0}\right)= & -\frac{1}{\epsilon}+2 \log \left(\frac{x}{2}\right)-\log (2) \sum_{m, n=1}^{\infty}\left(\frac{1}{2}\right)^{m} x^{n}\left(\begin{array}{c}
2 d_{0}-2+n \\
n
\end{array}\right) \\
& -\sum_{m, n=1}^{\infty}\left(\frac{x}{2}\right)^{m} x^{n}\left(\begin{array}{c}
2 d_{0}-2+m+n \\
m+n
\end{array}\right)\left[S_{1}\left(2 d_{0}-2+m+n\right)-S_{1}(m+n)+\log \left(\frac{x}{2}\right)\right],
\end{aligned}
$$

where the harmonic sums $S_{1}(n)$ are defined as [32, 33]

$$
S_{1}(n)=\sum_{i=1}^{n} \frac{1}{i}=\psi(n+1)+\gamma_{E},
$$

with $\gamma_{E}$ being Euler's constant and $\psi(x)$ being the polygamma function, i.e. the first derivative of the logarithm of the $\Gamma$-function. The first double sum amounts to

$$
\sum_{m, n=1}^{\infty}\left(\frac{1}{2}\right)^{m} x^{n}\left(\begin{array}{c}
2 d_{0}-2+n \\
n
\end{array}\right)=-\left(1-\frac{1}{(1-x)^{2 d_{0}-1}}\right) .
$$

We are not able to perform the second summation for arbitrary $d_{0}$. However, choosing integer values, $d_{0} \geq 2$, these sums simplify significantly. Indeed, if $d_{0}$ is a positive integer, then both

$$
\left(\begin{array}{c}
2 d_{0}-2+m+n \\
m+n
\end{array}\right), \quad\left(\begin{array}{c}
2 d_{0}-2+m+n \\
m+n
\end{array}\right)\left[S_{1}\left(2 d_{0}-2+m+n\right)-S_{1}(m+n)\right],
$$

are polynomials in $m$ and $n$. This implies that the double sums in eq. (3.13) can be written in terms of the functions

$$
\sum_{n=1}^{\infty} \frac{x^{n}}{n^{k}}=\left\{\begin{array}{cc}
\operatorname{Li}_{k}(x) & \text { if } k \geq 0, \\
\frac{1}{(1-x)^{1-k}} \sum_{i=0}^{-k-1}\left\langle\begin{array}{c}
-k \\
i
\end{array}\right\rangle x^{-k-i} & \text { if } k<0,
\end{array}\right.
$$

where $\operatorname{Li}_{k}(x)$ are the classical polylogarithms [38] and $\left\langle\begin{array}{c}-k \\ i\end{array}\right\rangle$ are the Eulerian numbers:

$$
\left\langle\begin{array}{c}
-k \\
i
\end{array}\right\rangle=\sum_{j=0}^{i+1}(-1)^{j}\left(\begin{array}{c}
-k+1 \\
j
\end{array}\right)(i-j+1)^{-k} ; \quad k<0 .
$$


Therefore, eq. (3.13) becomes for example with the choice $d_{0}=2$,

$$
\begin{aligned}
\mathcal{E}\left(x ; \epsilon, d_{0}\right)= & -\frac{1}{\epsilon}+\log (2)\left(1-\frac{1}{(1-x)^{3}}\right)-\frac{x^{2}\left(3 x^{2}-15 x+14\right)}{2(1-x)^{2}(2-x)^{2}} \\
& +\frac{\left(x^{6}-9 x^{5}+33 x^{4}-78 x^{3}+108 x^{2}-72 x+16\right)}{(1-x)^{3}(2-x)^{3}} \log \left(\frac{x}{2}\right)+\mathrm{O}(\epsilon) .
\end{aligned}
$$

Looking at this expression we notice that even if the integral in eq. (3.6) is well defined for $x=1$ or $x=2$ some of its individual terms diverge in these limits. Nevertheless, the full result has a well defined limit in $x=1$ or $x=2$. Indeed,

$$
\begin{aligned}
& \lim _{x \rightarrow 1} \mathcal{E}\left(x ; \epsilon, d_{0}\right)=-\frac{1}{\epsilon}+\frac{53}{6}-16 \log (2)+\mathrm{O}(\epsilon), \\
& \lim _{x \rightarrow 2} \mathcal{E}\left(x ; \epsilon, d_{0}\right)=-\frac{1}{\epsilon}-\frac{8}{3}+2 \log (2)+\mathrm{O}(\epsilon) .
\end{aligned}
$$

This completes the discussion of our example and demonstrates that the Laurent coefficients are given by simple functions in $x$ only. Looking back at how eq. (3.1) has enabled us to arrive at eq. (3.7) starting from eq. (3.6) it is obvious that more complicated integrals such as nested ones defined in section 2.2 result in increased numbers of Mellin integrations and shifted arguments of the $\Gamma$-functions. Also, in the case of nested integrals the order of the singularities is higher. However, the extraction of the poles in $\epsilon$ always reduces the dimensionality of the MB integrals (in our example from two to zero). Hence, in general if we start from a high-dimensional MB representation, the contributions to the poles' coefficients have a much lower dimensionality of the Mellin integrals, which allows for an analytic computation of the coefficients of the poles in the $\epsilon$ expansion even for the most complicated integrals. This example also shows that for the analytic computation by means of a MB representation one should choose $d_{0}, d_{0}^{\prime}$ to be positive integers and transform the regions of integrations in the integrals defined in section 2 to $[0,1]$. In this work we simply choose $\alpha_{0}=y_{0}=1$ and consider the cases $d_{0}=d_{0}^{\prime}=2,3$ which as discussed in ref. [22] are the natural choices for the infrared subtraction for processes with two and three outgoing jets respectively. Nevertheless, we stress that in principle any choice of $d_{0}, d_{0}^{\prime} \geq 2$ can be used in a computation of $m$-jet production, for any $m$. Thus there is no need to recompute any integrals even for processes with more than three jets. Furthermore, the appearance of a hypergeometric function in the integrand of eq. (3.6) as happens for example in the last row of table 1 does not essentially change the complexity of the computation. The reason is that the hypergeometric function ${ }_{2} F_{1}$ has a simple $\mathrm{MB}$ representation:

$$
{ }_{2} F_{1}(a, b, c ; w)=\int_{q-\mathrm{i} \infty}^{q+\mathrm{i} \infty} \frac{\mathrm{d} z}{2 \pi i}(-w)^{z} \Gamma\left(\begin{array}{c}
c, a+z, b+z,-z \\
a, b, c+z
\end{array}\right),
$$

where the integration contour separates the poles of the $\Gamma(\cdots+z)$ functions from the poles of the $\Gamma(\cdots-z)$ function as usual.

In closing this section, we would like to mention another virtue of the MB method. For a given phase space integral of section 2, the corresponding MB representations show good 
convergence properties if evaluated numerically along the complex contours. Thus, the multidimensional numerical integration of MB integrals, such as in eq. (3.9) is straightforward with the help of the CUBA library [39], which provides an independent check. Moreover, it also presents a quick and reliable way of obtaining numerical results for the (smooth) $\mathrm{O}\left(\epsilon^{0}\right)$ terms in the Laurent expansions of all integrals for the real-virtual counterterms in the paper.

\section{Collinear integrals $\mathcal{I}$}

In this section, we show the analytic results for the collinear integrals defined in eq. (2.5) for which the case $\kappa=0$ is needed only for the first row in table 1 and $\kappa=1$ is needed for all of them. Analytic expressions for the first two cases of table 1 have already been computed in ref. [21]. Here we fix $d_{0}=3$ and give the explicit expressions for this case as an illustration of the form of our results. For the Laurent expansion we obtain

$$
\begin{aligned}
\mathcal{I}\left(x ; \epsilon ; \alpha_{0}=1, d_{0} ; \kappa, k, \delta, g_{I}^{( \pm)}\right)= & \frac{\delta_{k,-1}}{2(2-\delta)} \frac{1}{\epsilon^{2}}-\left[\frac{2 \delta_{k,-1} \log (x)}{3-\delta}+\frac{1-\delta_{k,-1}}{2\left[1+k\left(1-\delta_{k,-1}\right)\right]}\right] \frac{1}{\epsilon} \\
& +\delta_{\kappa, 1} \mathcal{G}_{I, k}^{( \pm)}(x)+\mathcal{F}\left(x ; \epsilon, d_{0}, k\right)+\mathrm{O}(\epsilon)
\end{aligned}
$$

where $I=A, B, C, D$ (see table 1 ), $k=-1,0,1,2$ and $\delta_{i, j}$ is the usual Kronecker $\delta$. Here we have introduced the two functions $\mathcal{G}_{I, k}^{( \pm)}(x)$ and $\mathcal{F}\left(x ; \epsilon, d_{0}, k\right)$. The function $\mathcal{G}_{I, k}^{( \pm)}$is a matrix in $I$ (rows) and $k$ (columns) defined as follows:

$$
\mathcal{G}_{I, k}^{( \pm)}(x)=\left(\begin{array}{cccc}
\frac{2}{3} \zeta_{2}+\frac{1}{3} \log ^{2}(x) & 1 & \frac{1}{2} & \frac{13}{36} \\
\left(\frac{5}{8} \pm \frac{5}{8}\right) \zeta_{2}+\left(\frac{1}{2} \mp \frac{1}{2}\right) \log ^{2}(x) & 1 & \frac{1}{2} \pm \frac{1}{4} & \frac{13}{16} \pm \frac{1}{4} \\
\left(\frac{2}{3} \pm \frac{1}{2}\right) \zeta_{2}+\frac{1}{3} \log ^{2}(x) & 1 \pm \frac{1}{2} & \frac{1}{2} \pm \frac{3}{8} & \frac{13}{36} \pm \frac{11}{36} \\
\left(\frac{13}{36} \mp \frac{1}{16}\right) \zeta_{2}+\left(\frac{1}{2} \pm \frac{1}{2}\right) \log ^{2}(x) & 1 \pm \frac{1}{2} & \frac{1}{2} \pm \frac{1}{8} & \frac{13}{36} \pm \frac{1}{18}
\end{array}\right)
$$

and choosing e.g. $d_{0}=3$ for the function $\mathcal{F}\left(x ; \epsilon, d_{0},-1\right)$, we obtain

$$
\begin{aligned}
\mathcal{F}\left(x ; \epsilon, d_{0}=3,-1\right)= & -\frac{3}{2} \zeta_{2}+\log ^{2}(x)-\frac{1}{24} P_{0,-1}^{(5)}(x ; 35,-133,188,-116,0,0) \\
& -\frac{1}{12} P_{1,-1}^{(5)}(x ; 25,-116,212,-192,96,0)-P_{2,-1}^{(5)}(x ; 1,-5,10,-10,5,2), \\
\mathcal{F}\left(x ; \epsilon, d_{0}=3,0\right)= & -\frac{1}{12} P_{0,0}^{(5)}(x ; 49,-193,281,-173,24,0)+P_{1,0}^{(5)}(x ; 1,-5,10,-10,5,2), \\
\mathcal{F}\left(x ; \epsilon, d_{0}=3,1\right)= & \frac{1}{2} \mathcal{F}\left(x ; \epsilon, d_{0}=3,0\right), \\
\mathcal{F}\left(x ; \epsilon, d_{0}=3,2\right)= & \frac{80 x}{3(2-x)^{6}} \log (2)+\frac{(1-x)^{6}}{36(2-x)^{6}} P_{0,2}^{(11)}(x ; 51,-861,6523,-29212, \\
& +\frac{(1-x)^{6}}{3(2-x)^{6}} P_{1,2}^{(11)}(x ; 1,-17,130,-590,1765,-3734,5748,-6360, \\
& 4880,-2480,784,-128) .
\end{aligned}
$$



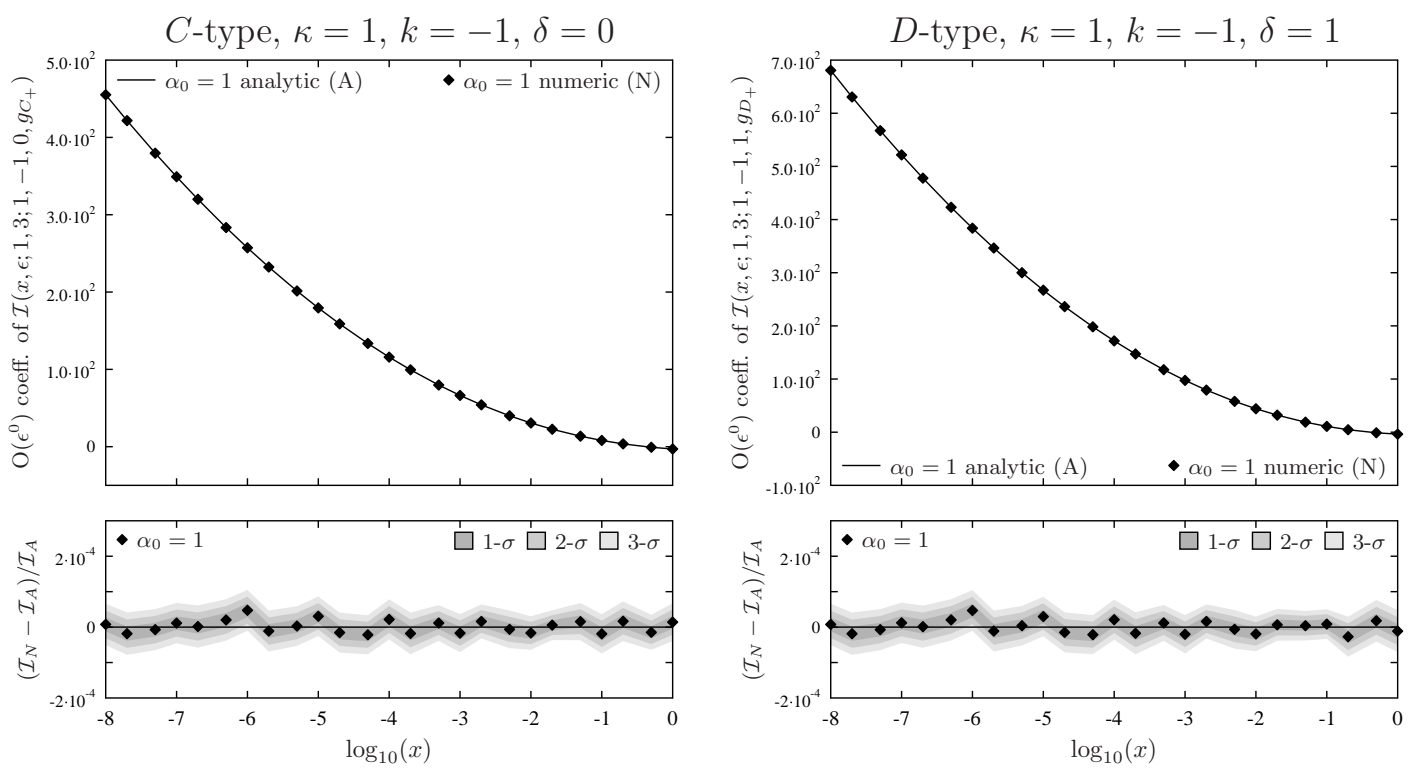

Figure 3. Representative results for the $C$-type and $D$-type integrals. The plots show the coefficient of the $\mathrm{O}\left(\epsilon^{0}\right)$ term for $k=-1$ in $\mathcal{I}\left(x, \epsilon ; 1,3 ; 1,-1,0, g_{C}^{(+)}\right)$(left) and $\mathcal{I}\left(x, \epsilon ; 1,3 ; 1,-1,1, g_{D}^{(+)}\right)$(right) with $d_{0}=3$ and $\alpha_{0}=1$.

Here we introduced the short-hand notation

$$
P_{n, k}^{(m)}\left(x ; a_{m}^{(k)}, \ldots, a_{0}^{(k)}\right)=\frac{\operatorname{Li}_{n}(1-x)}{(1-x)^{m}} \sum_{i=0}^{m} a_{i}^{(k)} x^{i} .
$$

According to their definition, the limit of the functions given in eqs. (4.3)-(4.6) must be finite in $x=1$ even if some terms are separately divergent. Indeed computing the limit at $x=1$ we find

$$
\begin{aligned}
\lim _{x \rightarrow 1} \mathcal{F}\left(x ; \epsilon, d_{0}=3,-1\right) & =-\frac{8731}{3600}-\frac{3}{2} \zeta_{2}, \\
\lim _{x \rightarrow 1} \mathcal{F}\left(x ; \epsilon, d_{0}=3,0\right) & =-\frac{257}{60}, \\
\lim _{x \rightarrow 1} \mathcal{F}\left(x ; \epsilon, d_{0}=3,1\right) & =-\frac{257}{120}, \\
\lim _{x \rightarrow 1} \mathcal{F}\left(x ; \epsilon, d_{0}=3,2\right) & =-\frac{1801}{90}+\frac{80}{3} \log (2) .
\end{aligned}
$$

In figure 3 we compare the analytic and numeric results for the $\epsilon^{0}$ coefficient in the expansion of $\mathcal{I}\left(x, \epsilon ; 1,3 ; 1,-1,0, g_{C}^{(+)}\right)$and $\mathcal{I}\left(x, \epsilon ; 1,3 ; 1,-1,1, g_{D}^{(+)}\right)$for $k=-1, \alpha_{0}=1$ and $d_{0}=3$ as representative examples. The agreement between the two computations is excellent for the whole $x$-range. The numeric results have been obtained using a sector decomposition [40] and Monte Carlo integration program as explained in detail in refs. [21, $22]$. This shows that the expansion coefficients of all the collinear integrals $\mathcal{I}$ and hence also of the collinear subtraction terms are smooth functions of the kinematical variable $x$. 
The complete results for all necessary cases (like in the later sections) are of considerable size, such that we shall not list them here. They are all contained in a MATHEMATICA file provided with the sources of the paper on the archive http://arXiv.org.

\section{$5 \quad$ Nested collinear-type $\mathcal{I} * \mathcal{I}$ and $\mathcal{I} * \mathcal{J}$ integrals}

In this section we discuss the analytic computation of the nested collinear integrals defined in eqs. (2.12)-(2.14).

As an example we show explicitly the fully analytic result for the case $\mathcal{I} *$ $\mathcal{I}_{r}(x, \epsilon ; 1,3 ;-1,2)$ for which we were able to compute the complete pole structure analytically. Choosing $d_{0}=3$ and $\alpha_{0}=1$ we get

$$
\begin{aligned}
\mathcal{I}_{*} \mathcal{I}_{r}(x, \epsilon ; 1,3 ;-1,2)=-\frac{1}{12} \frac{1}{\epsilon^{3}}+\left(-\frac{2}{9}+\frac{1}{3} \log (x)\right) \frac{1}{\epsilon^{2}}+\left[\frac { 1 } { ( 1 - x ) ^ { 5 } } \left(-\frac{1}{3} \zeta_{2}-\frac{25}{36} \log (x)\right.\right. \\
\left.+\frac{1}{3} \log (1-x) \log (x)+\frac{1}{3} \operatorname{Li}_{2}(x)\right)+\frac{1}{(1-x / 2)^{5}}\left(\frac{1}{6} \log \left(\frac{x}{2}\right)\right)+\frac{1}{(1-x)^{4}}\left(-\frac{13}{36}+\frac{1}{6} \log (x)\right) \\
+\frac{1 / 6}{(1-x / 2)^{4}}+\frac{1}{(1-x)^{3}}\left(-\frac{7}{72}-\frac{1}{18} \log (x)\right)+\frac{1 / 12}{(1-x / 2)^{3}}+\frac{1}{(1-x)^{2}}\left(-\frac{1}{6}-\frac{2}{9} \log (x)\right) \\
+\frac{1 / 18}{(1-x / 2)^{2}}+\frac{1}{(1-x)}\left(-\frac{25}{72}-\frac{7}{12} \log (x)\right)+\frac{1 / 24}{(1-x / 2)}+\frac{31}{216}+\frac{1}{6} \log (2) \\
\left.+\frac{19}{9} \log (x)+\frac{2}{3} \log (1-x) \log (x)-\frac{2}{3} \log ^{2}(x)+\frac{2}{3} \operatorname{Li}_{2}(x)\right] \frac{1}{\epsilon}+\mathrm{O}\left(\epsilon^{0}\right) .
\end{aligned}
$$

This result is representative, because its form is typical of all the collinear nested integrals. The plot of the $\mathrm{O}\left(\epsilon^{-1}\right)$ coefficient of this Laurent expansion for the integral $\mathcal{I} * \mathcal{I}_{r}(x, \epsilon ; 1,3 ;-1,2)$ is shown on the right side of figure 4 together with the comparison with the numerical evaluation obtained using sector decomposition and Monte Carlo integration. On the left side of figure 4 we plot the same coefficient of the Laurent expansion for the integral $\mathcal{I}_{*} \mathcal{I}_{i}(x, \epsilon ; 1,3 ;-1,2)$. For both cases we note that the agreement between the numerical evaluation and the analytic result is excellent. These plots show also that the coefficients of the Laurent expansion of the nested collinear integrals $\mathcal{I} * \mathcal{I}$ are very smooth functions of $x$.

We note that in the Laurent expansion of $\mathcal{I}_{*} \mathcal{I}_{r}(x, \epsilon ; 1,3 ;-1,2)$ in eq. (5.1) there are some terms that are divergent in $x=1$. However according to its definition in eq. (2.13) the limit in $x=1$ must be finite. To verify this is a further check of the correctness of the result. For the case of eq. (5.1) we obtain that:

$$
\lim _{x \rightarrow 1} \mathcal{I}_{* \mathcal{I}_{r}}(x, \epsilon ; 1,3 ;-1,2)=-\frac{1}{12} \frac{1}{\epsilon^{3}}-\frac{2}{9} \frac{1}{\epsilon^{2}}+\left(\frac{3091}{675}+\frac{2}{3} \zeta_{2}-\frac{31}{6} \log (2)\right) \frac{1}{\epsilon}+\mathrm{O}\left(\epsilon^{0}\right) .
$$

The case of $\mathcal{I}_{*} \mathcal{I}_{r}(x, \epsilon ; 1,3 ; 2,-1)$ is more difficult. For this integral we are unable to compute the coefficients of the $\epsilon$ poles in a fully analytic form. The reason is that in its Mellin-Barnes representation also three-fold MB integrals are involved. For this case the coefficient $\mathrm{O}\left(\epsilon^{-3}\right)$ and $\mathrm{O}\left(\epsilon^{-2}\right)$ are fully analytic but the coefficient of $\mathrm{O}\left(\epsilon^{-1}\right)$ is semi-analytic. This last coefficient is thus written in terms of an analytic expression 

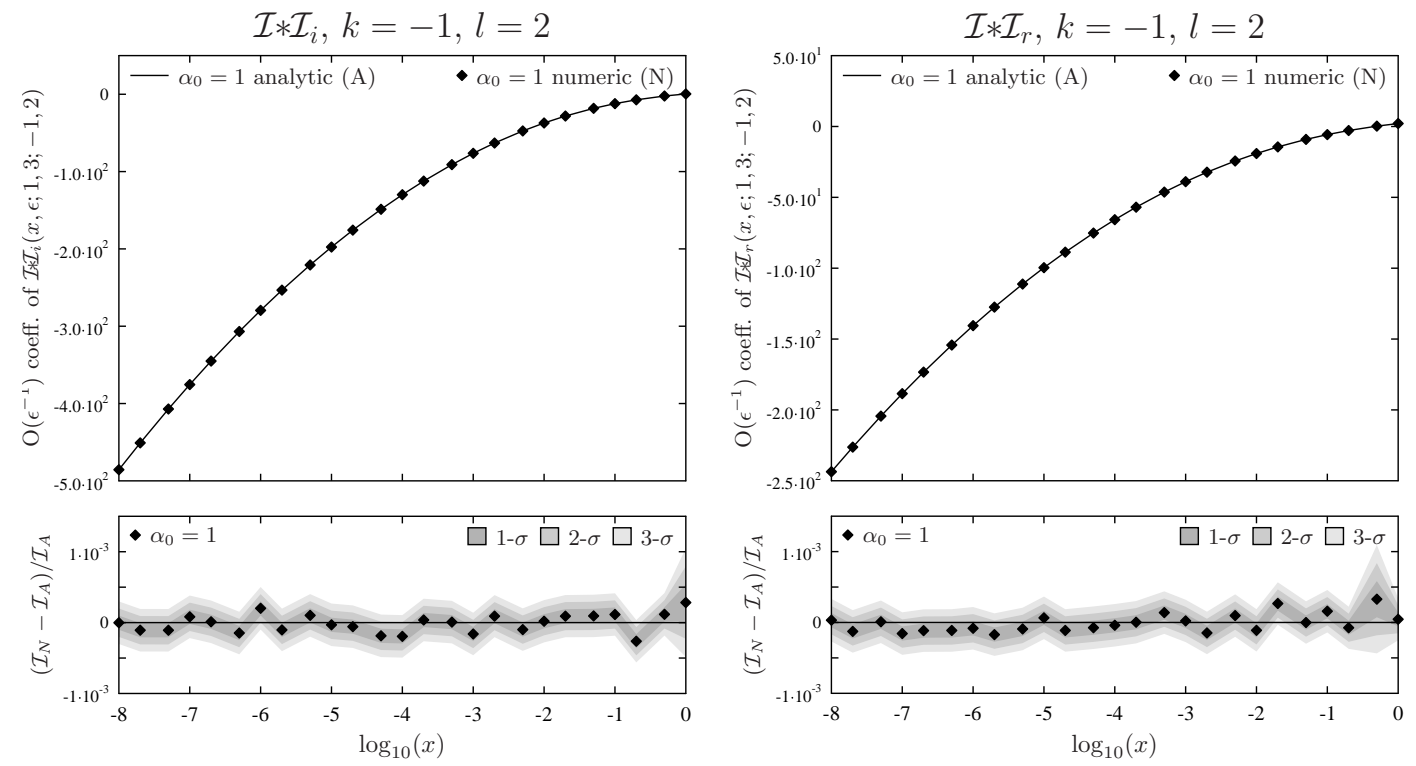

Figure 4. Representative results for the $\mathcal{I} * \mathcal{I}$-type integrals. The plots show the coefficient of the $\mathrm{O}\left(\epsilon^{-1}\right)$ term for $k=-1$ and $l=2$ in $\mathcal{I}_{*} \mathcal{I}_{i}(x, \epsilon ; 1,3 ;-1,2)$ (left) and $\mathcal{I}_{* \mathcal{I}_{r}}(x, \epsilon ; 1,3 ;-1,2)$ (right) with $d_{0}=3$ and $\alpha_{0}=1$.

to which a three-fold MB integral must be added. The remaining MB integral can be efficiently computed in MATHEMATICA by use of the package MB.m [29]. Explicitly for $\mathcal{I}$ * $\mathcal{I}_{r}(x, \epsilon ; 1,3 ; 2,-1)$ we have:

$$
\begin{aligned}
\mathcal{I}_{*} \mathcal{I}_{r}(x, \epsilon ; 1,3 ; 2,-1)=-\frac{1}{6} \frac{1}{\epsilon^{3}}+\left[\frac{1}{(1-x / 2)^{6}}\left(-\frac{5}{12} \log \left(\frac{x}{2}\right)\right)+\frac{1}{(1-x)^{5}}\left(\frac{1}{6} \log (x)\right)\right. \\
+\frac{1}{(1-x / 2)^{5}}\left(-\frac{5}{12}+\frac{5}{12} \log \left(\frac{x}{2}\right)\right)+\frac{1 / 6}{(1-x)^{4}}+\frac{5 / 24}{(1-x / 2)^{4}}+\frac{1 / 12}{(1-x)^{3}} \\
\left.+\frac{5 / 72}{(1-x / 2)^{3}}+\frac{1 / 18}{(1-x)^{2}}+\frac{5 / 144}{(1-x / 2)^{2}}+\frac{1 / 24}{(1-x)}+\frac{1 / 48}{(1-x / 2)}-\frac{59}{72}+\frac{1}{2} \log (x)\right] \frac{1}{\epsilon^{2}} \\
+\left[\frac { 1 } { ( 1 - x / 2 ) ^ { 6 } } \left(\frac{25}{24} \zeta_{2}-\frac{21}{8} \log (2)+\frac{5}{4} \log ^{2}(2)+\frac{5}{3} \log (2) \log (1-x / 2)+\frac{21}{8} \log (x)\right.\right. \\
-\frac{5}{2} \log (2) \log (x)+\frac{5}{12} \log (1-x) \log (x)-\frac{5}{3} \log (1-x / 2) \log (x)+\frac{5}{4} \log ^{2}(x) \\
\left.-\frac{5}{3} \operatorname{Li}_{2}\left(\frac{x}{2}\right)+\frac{5}{12} \operatorname{Li} 2(x)\right)+\frac{1}{(1-x)^{5}}\left(-\frac{1}{3} \zeta_{2}-\frac{1}{6} \log ^{2}(2)-\frac{1}{3} \log (2) \log (1-x / 2)\right. \\
+\frac{17}{24} \log (x)+\frac{1}{3} \log (2) \log (x)+\frac{1}{6} \log (1-x) \log (x)+\frac{1}{3} \log (1-x / 2) \log (x) \\
\left.-\frac{1}{2} \log ^{2}(x)+\frac{1}{3} \operatorname{Li} 2\left(\frac{x}{2}\right)+\frac{1}{6} \operatorname{Li}_{2}(x)\right)+\frac{1}{(1-x / 2)^{5}}\left(\frac{23}{24}-\frac{25}{24} \zeta_{2}+\frac{71}{24} \log (2)\right. \\
-\frac{5}{4} \log ^{2}(2)-\frac{5}{3} \log (2) \log (1-x / 2)-\frac{17}{8} \log (x)+\frac{5}{2} \log (2) \log (x)+ \\
\left.-\frac{5}{12} \log (1-x) \log (x)+\frac{5}{3} \log \left(1-\frac{x}{2}\right) \log (x)-\frac{5}{4} \log ^{2}(x)+\frac{5}{3} \operatorname{Li}_{2}\left(\frac{x}{2}\right)-\frac{5}{12} \operatorname{Li}_{2}(x)\right)
\end{aligned}
$$




$$
\begin{aligned}
& +\frac{1}{(1-x)^{4}}\left(\frac{7}{8}-\frac{1}{4} \zeta_{2}+\frac{2}{3} \log (2)-\frac{11}{8} \log (x)+\frac{1}{4} \log (1-x) \log (x)+\frac{1}{4} \operatorname{Li}_{2}(x)\right) \\
& +\frac{1}{(1-x / 2)^{4}}\left(-\frac{59}{48}+\frac{1}{6} \log \left(\frac{x}{2}\right)\right)+\frac{1}{(1-x)^{3}}\left(-\frac{1}{16}-\frac{1}{12} \zeta_{2}-\frac{7}{36} \log (x)\right. \\
& \left.+\frac{1}{12} \log (1-x) \log (x)+\frac{1}{12} \operatorname{Li}_{2}(x)\right)+\frac{1}{(1-x / 2)^{3}}\left(-\frac{29}{432}-\frac{1}{6} \log (2)+\frac{4}{9} \log (x)\right) \\
& +\frac{1}{(1-x)^{2}}\left(-\frac{1}{27}+\frac{2}{9} \log (2)-\frac{23}{36} \log (x)\right)+\frac{1}{(1-x / 2)^{2}}\left(\frac{211}{864}-\frac{2}{9} \log (2)+\frac{7}{9} \log (x)\right) \\
& +\frac{1}{(1-x)}\left(-\frac{31}{72}-\frac{59}{24} \log (x)\right)+\frac{1}{(1-x / 2)}\left(\frac{139}{288}-\frac{1}{3} \log (2)+\frac{4}{3} \log (x)\right)-\frac{1177}{432}+\frac{7}{8} \zeta_{2} \\
& -\frac{1}{3} \log (2)+\frac{1}{6} \log { }^{2}(2)+\frac{1}{3} \log (2) \log \left(1-\frac{x}{2}\right)+\frac{71}{24} \log (x)-\frac{1}{3} \log (2) \log (x) \\
& \left.+\frac{1}{2} \log (1-x) \log (x)-\frac{1}{3} \log \left(1-\frac{x}{2}\right) \log (x)-\frac{5}{6} \log ^{2}(x)\right)-\frac{1}{3} \operatorname{Li}\left(\frac{x}{2}\right)+\frac{1}{2} \operatorname{Li}_{2}(x) \\
& +\operatorname{MBint}[x]] \frac{1}{\epsilon}+\mathrm{O}\left(\epsilon^{0}\right),
\end{aligned}
$$

where MBint $[x]$ is a three-fold Mellin-Barnes integral, which for this case is given by

$$
\begin{aligned}
\operatorname{MBint}[x]= & \int_{q_{1}-\mathrm{i} \infty}^{q_{1}+\mathrm{i} \infty} \frac{\mathrm{d} z_{1}}{2 \pi i} \int_{q_{2}-\mathrm{i} \infty}^{q_{2}+\mathrm{i} \infty} \frac{\mathrm{d} z_{2}}{2 \pi i} \int_{q_{3}-\mathrm{i} \infty}^{q_{3}+\mathrm{i} \infty} \frac{\mathrm{d} z_{3}}{2 \pi i} 2^{z_{3}-1} x^{-z_{1}-z_{2}-z_{3}} \\
& \times \Gamma\left(\begin{array}{c}
-z_{1}, 1+z_{1}, 3-z_{2},-2+z_{2}, 5-z_{1}-z_{2}-z_{3},-z_{3}, 2+z_{3}, z_{1}+z_{2}+z_{3} \\
4,4-z_{2}
\end{array}\right),
\end{aligned}
$$

where $q_{1}=q_{2}=q_{3}=-1 / 4$.

Similarly to the analytic expression of eq. (5.1), also in this case we have many terms that are singular in $x=1$ even though the full expression is well defined. Moreover in cases like this where we have a semi-analytic expression we find that the analytic part and the remaining part expressed in terms of a three-fold MB integral are separately well defined in $x=1$. In particular for the case of the integral $\mathcal{I}_{*} \mathcal{I}_{r}(x, \epsilon ; 1,3 ; 2,-1)$ in eq. (5.3) we obtain the following limit:

$$
\begin{aligned}
\lim _{x \rightarrow 1} \mathcal{I}_{*} \mathcal{I}_{r}(x, \epsilon ; 1,3 ; 2,-1)= & -\frac{1}{6} \frac{1}{\epsilon^{3}}+\left(-\frac{607}{60}+\frac{40}{3} \log (2)\right) \frac{1}{\epsilon^{2}}+\left(\frac{77349}{14400}+\frac{509}{24} \zeta_{2}\right. \\
& \left.-\frac{3571}{45} \log (2)+\frac{40}{3} \log ^{2}(2)+\operatorname{MBint}[1]\right) \frac{1}{\epsilon}+\mathrm{O}\left(\epsilon^{0}\right),
\end{aligned}
$$

where MBint[1] is given by

$$
\text { MBint[1] }=0.329808 .
$$

This number is the result of the MB integral in eq. (5.4) with the choice $x=1$ obtained using the MATHEMATICA package MB.m [29]. Finally we note that this example is representative for a small subset of the collinear nested integrals which have these features. They are $\mathcal{I} *$ $\mathcal{I}_{i}(x, \epsilon ; 1,3 ; k, l)$ and $\mathcal{I}_{*} \mathcal{I}_{r}(x, \epsilon ; 1,3 ; k, l)$ with $k=-1,1,2$ and $l=-1$ and $\mathcal{I}_{*} \mathcal{J}(x, \epsilon ; 1,3,1,3 ; k)$ with $k=-1$. The results for the pole structure of all the remaining cases of nested collinear integrals are fully analytic. 


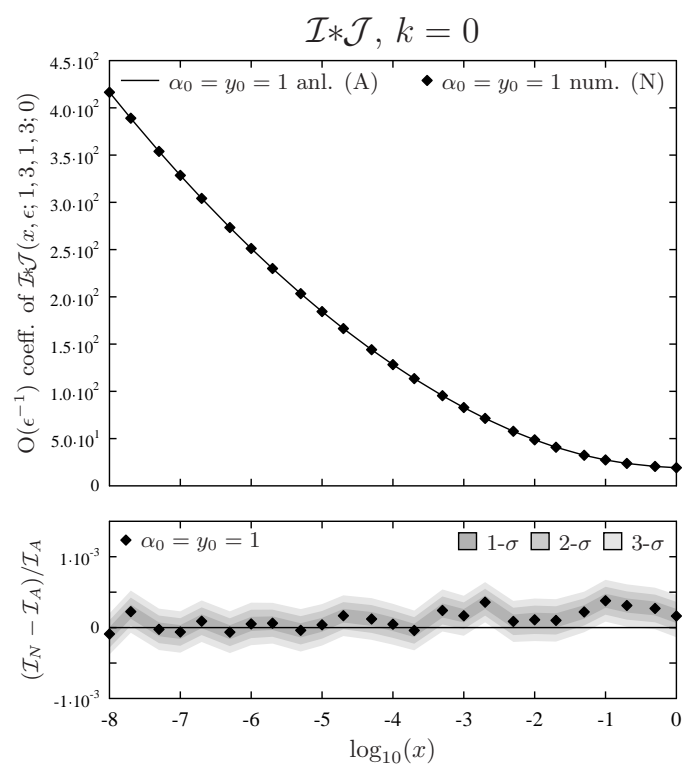

Figure 5. Representative results for the $\mathcal{I} * \mathcal{J}$-type integrals. The plots show the coefficient of the $\mathrm{O}\left(\epsilon^{-1}\right)$ term for $k=0$ in $\mathcal{I} * \mathcal{J}(x, \epsilon ; 1,3,1,3 ; 0)$ with $d_{0}=d_{0}^{\prime}=3$ and $\alpha_{0}=y_{0}=1$.

In table 2 we list numerical values for the non-trivial coefficients of the $\epsilon$-poles (i.e. the $\mathrm{O}\left(\epsilon^{-2}\right)$ and $\mathrm{O}\left(\epsilon^{-1}\right)$ coefficients) of the nested collinear integrals $\mathcal{I}_{*} \mathcal{I}_{r}(x, \epsilon ; 1,3 ;-1,2)$ and $\mathcal{I}_{*} \mathcal{I}_{r}(x, \epsilon ; 1,3 ; 2,-1)$. These numbers have been obtained using the fully analytic expression in eq. (5.1) and the semi-analytic one in eq. (5.3). For this last case using the default options of numerical integration accuracy in MB.m the relative uncertainty is at most of order $10^{-5}$, for values of $x$ around one. For $x \ll 1$, we find that the analytic part of the full semi-analytic result contains all contributions that are divergent as $x \rightarrow 0$ and in fact the numeric contribution decreases as we approach the limit. Thus the relative uncertainties become very small. Numbers for the $\mathrm{O}\left(\epsilon^{0}\right)$ coefficient for the same representative integrals are listed in table 3 . In this case they have been entirely obtained evaluating their $\mathrm{MB}$ representations. The relative uncertainties reported in table 3 were obtained with the numerical integration option MaxPoints set to $5 \cdot 10^{7}$ in MB.m.

Finally in figure 5 we plot as a further example the fully analytic result for the first order $\epsilon$-pole for $\mathcal{I} * \mathcal{J}(x, \epsilon ; 1,3 ; 0)$ together with the numbers obtained numerically using sector decomposition and Monte Carlo integration. As for all other cases the agreement is excellent and the coefficient is given by a very smooth function of $x$.

In this section as in the rest of the paper the representative plots are shown for the most complicated integrals for which a full analytic result was obtained.

\section{$6 \quad$ Nested soft-type $\mathcal{J} * \mathcal{J}$ integrals}

In this section we discuss the analytic computation of the integrals defined in eqs. (2.15)-(2.17). For them we were able to compute a fully analytic result for the coefficient of the Laurent expansion up to $\mathrm{O}\left(\epsilon^{-2}\right)$. The $\mathrm{O}\left(\epsilon^{-1}\right)$ coefficient is computed 


\begin{tabular}{|c|c|c|c|c|}
\hline $\log _{10}(x)$ & \multicolumn{2}{|c|}{$\mathcal{I}_{*} \mathcal{I}_{r}(x, \epsilon ; 1,3 ;-1,2)$} & \multicolumn{2}{|c|}{$\mathcal{I} * \mathcal{I}_{r}(x, \epsilon ; 1,3 ; 2,-1)$} \\
\hline & $\mathrm{O}\left(\epsilon^{-2}\right)$ an. & $\mathrm{O}\left(\epsilon^{-1}\right)$ an. & $\mathrm{O}\left(\epsilon^{-2}\right)$ an. & $\mathrm{O}\left(\epsilon^{-1}\right)$ semi-an. \\
\hline-10. & -7.89751 & -374.957 & -15.9061 & $-759.736 \pm 2.16974 \mathrm{E}-12$ \\
\hline-9.66667 & -7.64166 & -351.104 & -15.3944 & $-711.688 \pm 2.92666 \mathrm{E}-12$ \\
\hline-9.33333 & -7.38582 & -328.036 & -14.8828 & $-665.211 \pm 4.09941 \mathrm{E}-12$ \\
\hline-9. & -7.12998 & -305.753 & -14.3711 & $-620.305 \pm 6.01115 \mathrm{E}-12$ \\
\hline-8.66667 & -6.87413 & -284.256 & -13.8594 & $-576.969 \pm 7.79755 \mathrm{E}-12$ \\
\hline-8.33333 & -6.61829 & -263.544 & -13.3477 & $-535.205 \pm 1.08953 \mathrm{E}-11$ \\
\hline-8. & -6.36245 & -243.618 & -12.836 & $-495.012 \pm 1.54886 \mathrm{E}-11$ \\
\hline-7.66667 & -6.10661 & -224.477 & -12.3243 & $-456.389 \pm 1.84206 \mathrm{E}-11$ \\
\hline-7.33333 & -5.85076 & -206.122 & -11.8126 & $-419.337 \pm 2.91762 \mathrm{E}-11$ \\
\hline-7. & -5.59492 & -188.552 & -11.301 & $-383.857 \pm 3.7663 \mathrm{E}-11$ \\
\hline-6.66667 & -5.33908 & -171.768 & -10.7893 & $-349.947 \pm 4.44314 \mathrm{E}-11$ \\
\hline-6.33333 & -5.08324 & -155.769 & -10.2776 & $-317.608 \pm 6.83357 \mathrm{E}-11$ \\
\hline-6. & -4.82739 & -140.556 & -9.7659 & $-286.841 \pm 8.82413 \mathrm{E}-11$ \\
\hline-5.66667 & -4.57155 & -126.128 & -9.25423 & $-257.644 \pm 1.11892 \mathrm{E}-10$ \\
\hline-5.33333 & -4.31571 & -112.485 & -8.74256 & $-230.019 \pm 2.04546 \mathrm{E}-10$ \\
\hline-5. & -4.05986 & -99.628 & -8.2309 & $-203.965 \pm 4.4044 \mathrm{E}-10$ \\
\hline-4.66667 & -3.80402 & -87.556 & -7.71928 & $-179.482 \pm 9.48925 \mathrm{E}-10$ \\
\hline-4.33333 & -3.54818 & -76.269 & -7.20772 & $-156.573 \pm 2.0463 \mathrm{E}-9$ \\
\hline-4 . & -3.29234 & -65.7665 & -6.69628 & $-135.237 \pm 4.4068 \mathrm{E}-9$ \\
\hline-3.66667 & -3.03649 & -56.0477 & -6.18508 & $-115.476 \pm 9.49612 \mathrm{E}-9$ \\
\hline-3.33333 & -2.78065 & -47.1111 & -5.6743 & $-97.2944 \pm 2.04532 \mathrm{E}-8$ \\
\hline-3. & -2.52481 & -38.9536 & -5.16432 & $-80.6957 \pm 4.39917 \mathrm{E}-8$ \\
\hline-2.66667 & -2.26896 & -31.5702 & -4.65576 & $-65.6862 \pm 9.46543 \mathrm{E}-8$ \\
\hline-2.33333 & -2.01312 & -24.9522 & -4.14969 & $-52.2723 \pm 2.03847 \mathrm{E}-7$ \\
\hline-2. & -1.75728 & -19.0853 & -3.64776 & $-40.4585 \pm 4.37447 \mathrm{E}-7$ \\
\hline-1.66667 & -1.50144 & -13.9478 & -3.15236 & $-30.2408 \pm 9.3859 \mathrm{E}-7$ \\
\hline-1.33333 & -1.24559 & -9.50936 & -2.66658 & $-21.5965 \pm 1.99232 \mathrm{E}-6$ \\
\hline-1. & -0.989751 & -5.73082 & -2.19382 & $-14.4712 \pm 4.20263 \mathrm{E}-6$ \\
\hline-0.666667 & -0.733908 & -2.5675 & -1.73699 & $-8.76877 \pm 8.68698 \mathrm{E}-6$ \\
\hline-0.333333 & -0.478065 & 0.0265877 & -1.2975 & $-4.35339 \pm 1.73005 \mathrm{E}-5$ \\
\hline 0. & -0.222222 & 2.09462 & -0.874704 & $-1.06702 \pm 3.28582 \mathrm{E}-5$ \\
\hline
\end{tabular}

Table 2. Numerical values for the $\mathrm{O}\left(\epsilon^{-2}\right)$ and $\mathrm{O}\left(\epsilon^{-1}\right)$ coefficients of $\mathcal{I}_{*} \mathcal{I}_{r}(x, \epsilon ; 1,3 ;-1,2)$ (second and third column) and $\mathcal{I}_{*} \mathcal{I}_{r}\left(x, \epsilon ; 1,3 ; 2,-1\right.$ ) (last two columns) for various values of $\log _{10}(x)$ (first column). These numbers have been obtained evaluating the fully analytic expression in eq. (5.1) and the semi-analytic one in eq. (5.3). In the last column, we also show the numerical uncertainty as reported by MB.m. 


\begin{tabular}{|l|c|c|}
\hline $\log _{10}(x)$ & $\mathcal{I}_{*} \mathcal{I}_{r}(x, \epsilon ; 1,3 ;-1,2)$ & $\mathcal{I}_{*} \mathcal{I}_{r}(x, \epsilon ; 1,3 ; 2,-1)$ \\
\hline-5. & $-1642.9 \pm 1.40321$ & $-3380.06 \pm 1.88480 \mathrm{E}-1$ \\
\hline-4.66667 & $-1355.91 \pm 2.483460 \mathrm{E}-1$ & $-2792.08 \pm 5.41150 \mathrm{E}-2$ \\
\hline-4.33333 & $-1104.52 \pm 4.46988 \mathrm{E}-2$ & $-2276.68 \pm 2.74382 \mathrm{E}-2$ \\
\hline-4. & $-886.641 \pm 1.16175 \mathrm{E}-2$ & $-1829.26 \pm 2.16858 \mathrm{E}-2$ \\
\hline-3.66667 & $-699.73 \pm 8.45731 \mathrm{E}-3$ & $-1444.98 \pm 1.81844 \mathrm{E}-2$ \\
\hline-3.33333 & $-541.355 \pm 7.21400 \mathrm{E}-3$ & $-1119.05 \pm 1.53613 \mathrm{E}-2$ \\
\hline-3. & $-409.039 \pm 5.95157 \mathrm{E}-3$ & $-846.659 \pm 1.28763 \mathrm{E}-2$ \\
\hline-2.66667 & $-300.296 \pm 4.91329 \mathrm{E}-3$ & $-622.987 \pm 1.08469 \mathrm{E}-2$ \\
\hline-2.33333 & $-212.588 \pm 3.96846 \mathrm{E}-3$ & $-443.178 \pm 8.21964 \mathrm{E}-3$ \\
\hline-2. & $-143.342 \pm 3.13593 \mathrm{E}-3$ & $-302.315 \pm 6.39871 \mathrm{E}-3$ \\
\hline-1.66667 & $-89.9695 \pm 2.39827 \mathrm{E}-3$ & $-195.382 \pm 4.76177 \mathrm{E}-3$ \\
\hline-1.33333 & $-49.9198 \pm 1.77836-3$ & $-117.264 \pm 3.27515 \mathrm{E}-3$ \\
\hline-1. & $-20.7586 \pm 1.13024 \mathrm{E}-3$ & $-62.7773 \pm 2.08013 \mathrm{E}-3$ \\
\hline-0.666667 & $-0.267976 \pm 6.05459 \mathrm{E}-4$ & $-26.8568 \pm 1.13023 \mathrm{E}-3$ \\
\hline-0.333333 & $13.489 \pm 3.05477 \mathrm{E}-4$ & $-4.81243 \pm 5.52177 \mathrm{E}-4$ \\
\hline 0. & $22.1524 \pm 5.24078 \mathrm{E}-4$ & $7.37746 \pm 4.201149 \mathrm{E}-4$ \\
\hline
\end{tabular}

Table 3. Numerical values for the $\mathrm{O}\left(\epsilon^{0}\right)$ coefficient of $\mathcal{I}_{*} \mathcal{I}_{r}(x, \epsilon ; 1,3 ;-1,2)$ (second column) and I $\mathcal{I}_{r}(x, \epsilon ; 1,3 ; 2,-1)$ (last column) for various values of $\log _{10}(x)$ (first column). These numbers have been obtained evaluating their MB representation. Aalso shown are the numerical uncertainties as reported by MB.m.

semi-analytically similarly to the nested collinear integral $\mathcal{I}_{*} \mathcal{I}_{r}(x, \epsilon ; 1,3 ; 2,-1)$ discussed in section 5. As a representative example we show the structure of the fully analytic part of the result for the nested soft integrals $\mathcal{J} * \mathcal{J}$. For example choosing $d_{0}^{\prime}=3$ we have:

$$
\begin{aligned}
\mathcal{J} * \mathcal{J}_{i k}(Y ; \epsilon ; 1,3)= & \frac{1}{\epsilon^{4}}+\left(\frac{22}{3}-2 \log (Y)\right) \frac{1}{\epsilon^{3}}+\mathcal{H}(Y) \frac{1}{\epsilon^{2}}+\mathrm{O}\left(\epsilon^{-1}\right), \\
\mathcal{J} * \mathcal{J}_{i r}(Y ; \epsilon ; 1,3)=\frac{1}{2} \frac{1}{\epsilon^{4}}+\left(\frac{11}{3}-\log (Y)\right) \frac{1}{\epsilon^{3}}+( & \frac{533}{36}-\frac{22}{3} \log (Y)+\log ^{2}(Y) \\
& \left.+\frac{3}{2} \operatorname{Li}_{2}(1-Y)\right) \frac{1}{\epsilon^{2}}+\mathrm{O}\left(\epsilon^{-1}\right)
\end{aligned}
$$

and finally

$$
\mathcal{J} * \mathcal{J}_{k r}(Y ; \epsilon ; 1,3)=\frac{1}{\epsilon^{4}}+\left(\frac{22}{3}-2 \log (Y)\right) \frac{1}{\epsilon^{3}}+\left(\mathcal{H}(Y)-\zeta_{2}+\frac{1}{2} \operatorname{Li}_{2}(1-Y)\right) \frac{1}{\epsilon^{2}}+\mathrm{O}\left(\epsilon^{-1}\right)
$$

The function $\mathcal{H}(Y)$ which appears in eqs. (6.1) and (6.3) is given by

$\mathcal{H}(Y)=\frac{497}{18}-2 \zeta_{2}+\frac{6-8 Y}{3(1-Y)^{2}}+\frac{33 Y^{3}-117 Y^{2}+126 Y-44}{3(1-Y)^{3}} \log (Y)+2 \log ^{2}(Y)+4 \operatorname{Li}_{2}(1-Y)$. 
Also for this function even if some terms are singular at $Y=1$, we still have that the limit is well defined. Indeed we find

$$
\lim _{Y \rightarrow 1} \mathcal{H}(Y)=\frac{97}{3}-2 \zeta_{2}
$$

For these three soft-type integrals the $\mathrm{O}\left(\epsilon^{-2}\right)$ coefficient has been plotted in figure 6 using its fully analytic expression eqs. (6.1) and (6.4) and its numerical evaluation obtained using sector decomposition and Monte Carlo integration. The agreement is excellent and the analytic result confirms that also the coefficients of the Laurent expansion for the $\mathcal{J} * \mathcal{J}$ integrals are smooth functions of $Y$.

The numbers in table 4 have been obtained evaluating the nested soft integral $\mathcal{J} * \mathcal{J}_{i k}(Y ; \epsilon ; 1,3)$ using the fully analytic expression in eq. (6.1) for the $\mathrm{O}\left(\epsilon^{-3}\right)$ and $\mathrm{O}\left(\epsilon^{-2}\right)$ coefficients. For the $\mathrm{O}\left(\epsilon^{-1}\right)$ coefficient a semi-analytic expression in terms of a MB integral has been used and finally the representation only in terms of MB integrals has been evaluated for the $\mathrm{O}\left(\epsilon^{0}\right)$ coefficient. In this example the relative uncertainty as reported by MB.m with the default options for numerical integration for both the $\mathrm{O}\left(\epsilon^{-1}\right)$ and $\mathrm{O}\left(\epsilon^{0}\right)$ coefficients is at most of order $10^{-5}$. For the semi-analyitc result, we see a similar phenomenon as in the case of the $\mathcal{I}_{*} \mathcal{I}_{r}(x, \epsilon ; 1,3 ; 2,-1)$ integral: in the $Y \rightarrow 0$ limit, the analytic part contains all divergent contributions while the numeric part decreases. The relative uncertainties thus become very small.

\section{$7 \quad$ Nested soft-collinear $\mathcal{K} * \mathcal{J}$ integral}

In this last section we discuss the pole structure of the integral defined in eq. (2.18). In this case the result for the Laurent expansion is very simple because the integral has no dependence on the kinematics. The coefficients of the poles in $\mathcal{K} * \mathcal{J}(\epsilon, 1,3)$ with $d_{0}^{\prime}=3$ and $y_{0}=1$ read:

$$
\mathcal{K} * \mathcal{J}(\epsilon, 1,3)=-\frac{1}{2} \frac{1}{\epsilon^{4}}-\frac{11}{3} \frac{1}{\epsilon^{3}}-\frac{557}{36} \frac{1}{\epsilon^{2}}+\left(-\frac{10825}{216}+\frac{5}{3} \zeta_{2}-3 \zeta_{3}\right) \frac{1}{\epsilon}+\mathrm{O}\left(\epsilon^{0}\right) .
$$

This completes our discussion of the analytic computation of the fundamental integrals that contribute to the singly-unresolved counterterms.

\section{Conclusions}

In this work we have completed the evaluation of all integrals needed for the computation of the integrated real-virtual counterterms of the subtraction scheme for NNLO jet cross sections proposed in refs. [17-19]. We have discussed representative examples for all types of soft and collinear as well as nested integrals in sections 4-7 (the complete results are contained in a MATHEMATICA file). These integrals (i.e. their Laurent expansions in $\epsilon$ to sufficient depth) have to be computed once and for all and their knowledge is necessary in order to make the subtraction scheme an effective tool. We have achieved this task by deriving $\mathrm{MB}$ representations for all integrals under consideration and, in a subsequent step, we have performed analytically the summation of the nested sums over the series 

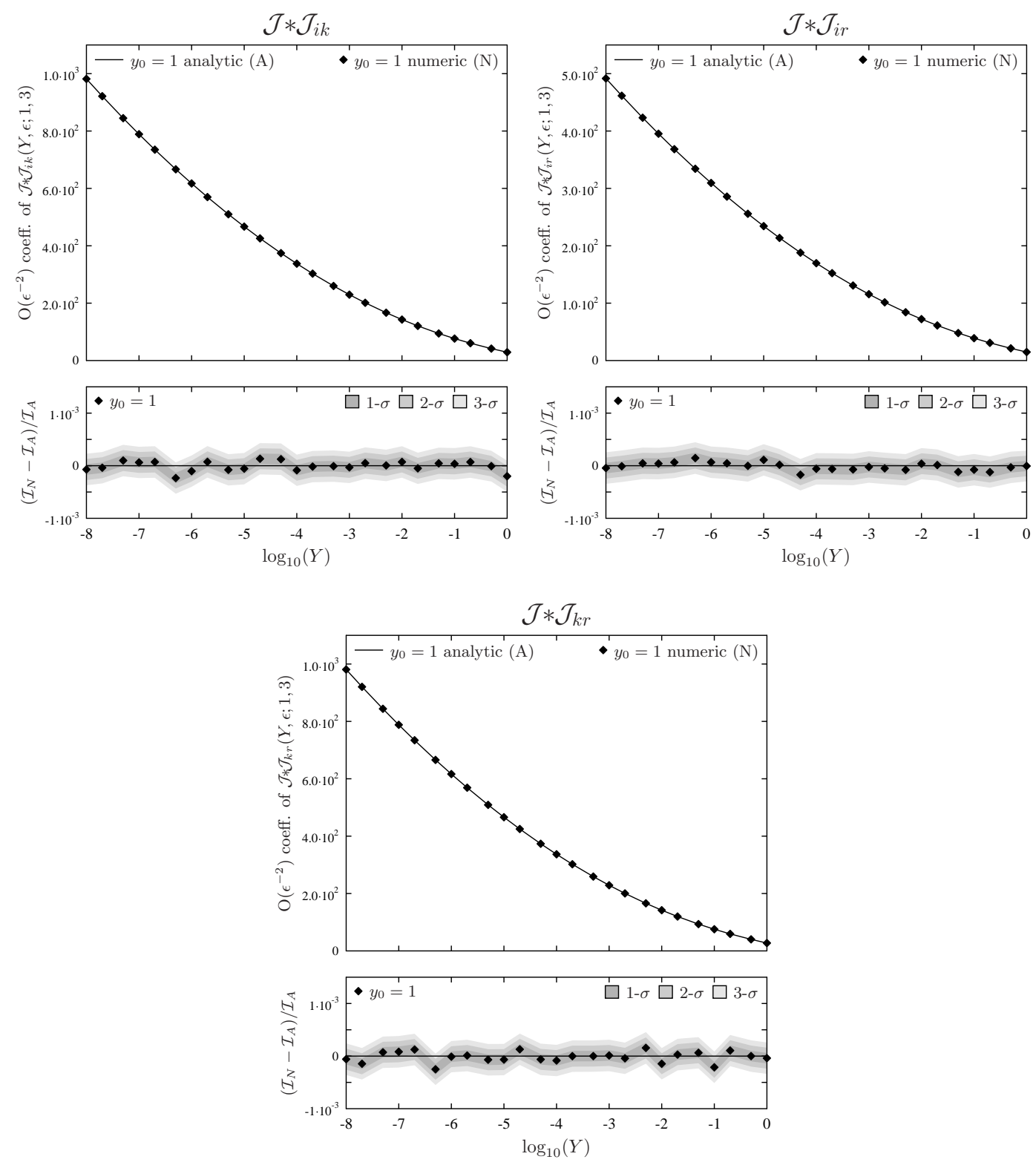

Figure 6. Representative results for the $\mathcal{J} * \mathcal{J}$-type integrals. The plots show the coefficient of the $\mathrm{O}\left(\epsilon^{-2}\right)$ term in $\mathcal{J} * \mathcal{J}_{i k}(Y, \epsilon ; 1,3)$ (left), $\mathcal{J} * \mathcal{J}_{i r}(Y, \epsilon ; 1,3)$ (right) and $\mathcal{J} * \mathcal{J}_{k r}(Y, \epsilon ; 1,3)$ (bottom) with $d_{0}^{\prime}=3$ and $y_{0}=1$.

of residues. In some cases, this second step of summing the series has not been achieved and we have resorted to a numerical evaluation of the MB integrals in the complex plane. All MB representations for both the numerical and, if available, the analytic results have been checked by an independent evaluation of the integrals using sector decomposition as in ref. [22]. We have shown, that all integrals contributing to the real-virtual counterterms are smooth functions. For practical applications, this means that all integrals (in particular the finite in $\epsilon$ contributions) can be used in terms of interpolating tables, which can be computed once and for all. Here we want to stress again that the tables and plots we 


\begin{tabular}{|c|c|c|c|c|}
\hline $\log _{10}(Y)$ & \multicolumn{4}{|c|}{$\mathcal{J} * \mathcal{J}_{i k}(Y ; \epsilon ; 1,3)$} \\
\hline & $\mathrm{O}\left(\epsilon^{-3}\right)$ an. & $\mathrm{O}\left(\epsilon^{-2}\right)$ an. & $\mathrm{O}\left(\epsilon^{-1}\right)$ semi-an. & $\mathrm{O}\left(\epsilon^{0}\right) \mathrm{MB}$ \\
\hline-10 & 53.385 & 1430.99 & 25680. $\pm 1.44332 \mathrm{E}-11$ & $347094 . \pm 1.69388 \mathrm{E}-3$ \\
\hline-9.66667 & 51.85 & 1350.22 & $23545.6 \pm 2.90334 \mathrm{E}-11$ & 309328. $\pm 1.31378 \mathrm{E}-3$ \\
\hline-9.33333 & 50.3149 & 1271.81 & $21533.4 \pm 5.83683 \mathrm{E}-11$ & $274744 . \pm 1.09995 \mathrm{E}-3$ \\
\hline-9. & 48.7799 & 1195.75 & $19639.8 \pm 1.17829 \mathrm{E}-10$ & 243157. $\pm 9.10225 \mathrm{E}-4$ \\
\hline-8.66667 & 47.2448 & 1122.05 & $17861.1 \pm 2.34891 \mathrm{E}-10$ & 214389. $\pm 9.47250 \mathrm{E}-4$ \\
\hline-8.33333 & 45.7098 & 1050.7 & $16193.8 \pm 4.73302 \mathrm{E}-10$ & 188265. $\pm 8.07442 \mathrm{E}-4$ \\
\hline-8 & 44.1747 & 981.714 & $14634.2 \pm 9.39828 \mathrm{E}-10$ & 164617. $\pm 6.22748 \mathrm{E}-4$ \\
\hline-7.66667 & 42.6396 & 915.081 & $13178.6 \pm 1.86537 \mathrm{E}-9$ & 143283. $\pm 5.50516 \mathrm{E}-4$ \\
\hline-7.33333 & 41.1046 & 850.805 & $11823.6 \pm 3.70809 \mathrm{E}-9$ & 124106. $\pm 5.03212 \mathrm{E}-4$ \\
\hline-7. & 39.5695 & 788.886 & $10565.3 \pm 7.23854 \mathrm{E}-9$ & 106934. $\pm 5.38078 \mathrm{E}-4$ \\
\hline-6.66667 & 38.0345 & 729.322 & $9400.38 \pm 1.44201 \mathrm{E}-8$ & 91621. $\pm 5.52685 \mathrm{E}-4$ \\
\hline-6.33333 & 36.4994 & 672.116 & $8325.04 \pm 2.82069 \mathrm{E}-8$ & $78027.5 \pm 5.08049 \mathrm{E}-4$ \\
\hline-6 & 34.9644 & 617.265 & $7335.7 \pm 5.49916 \mathrm{E}-8$ & $66018.2 \pm 5.08676 \mathrm{E}-4$ \\
\hline-5.66667 & 33.4293 & 564.771 & $6428.75 \pm 1.05801 \mathrm{E}-7$ & $55463.9 \pm 5.09976 \mathrm{E}-4$ \\
\hline-5.33333 & 31.8942 & 514.633 & $5600.58 \pm 2.03064 \mathrm{E}-7$ & $46240.8 \pm 4.63345 \mathrm{E}-4$ \\
\hline-5. & 30.3592 & 466.852 & $4847.56 \pm 3.86389 \mathrm{E}-7$ & $38230.9 \pm 5.01881 \mathrm{E}-4$ \\
\hline-4.66667 & 28.8241 & 421.427 & $4166.08 \pm 7.45290 \mathrm{E}-7$ & $31321.6 \pm 6.62424 \mathrm{E}-4$ \\
\hline-4.33333 & 27.2891 & 378.358 & $3552.51 \pm 1.35799 \mathrm{E}-6$ & $25405.6 \pm 6.96993 \mathrm{E}-4$ \\
\hline-4. & 25.754 & 337.645 & $3003.24 \pm 2.52452 \mathrm{E}-6$ & $20381.7 \pm 7.53947 \mathrm{E}-4$ \\
\hline-3.66667 & 24.219 & 299.287 & $2514.63 \pm 4.73890 \mathrm{E}-6$ & $16153.6 \pm 7.61827 \mathrm{E}-4$ \\
\hline-3.33333 & 22.6839 & 263.283 & $2083.06 \pm 8.67357 \mathrm{E}-6$ & 12631. $\pm 8.70475 \mathrm{E}-4$ \\
\hline-3. & 21.1488 & 229.632 & $1704.89 \pm 1.59261 \mathrm{E}-5$ & $9728.88 \pm 8.12022 \mathrm{E}-4$ \\
\hline-2.66667 & 19.6138 & 198.33 & $1376.45 \pm 2.79802 \mathrm{E}-5$ & $7367.63 \pm 8.23619 \mathrm{E}-4$ \\
\hline-2.33333 & 18.0787 & 169.37 & $1094.05 \pm 4.80260 \mathrm{E}-5$ & $5473.13 \pm 9.09435 \mathrm{E}-4$ \\
\hline-2. & 16.5437 & 142.739 & $853.961 \pm 8.03383 \mathrm{E}-5$ & $3976.65 \pm 1.09059 \mathrm{E}-3$ \\
\hline-1.66667 & 15.0086 & 118.417 & $652.369 \pm 1.30870 \mathrm{E}-4$ & $2814.76 \pm 1.41898 \mathrm{E}-3$ \\
\hline-1.33333 & 13.4736 & 96.3641 & $485.392 \pm 2.06356 \mathrm{E}-4$ & $1929.49 \pm 1.90893 \mathrm{E}-3$ \\
\hline-1. & 11.9385 & 76.5204 & $349.046 \pm 3.17701 \mathrm{E}-4$ & $1268.34 \pm 2.67185 \mathrm{E}-3$ \\
\hline-0.666667 & 10.4034 & 58.7892 & $239.262 \pm 4.48758 \mathrm{E}-4$ & $784.581 \pm 3.65297 \mathrm{E}-3$ \\
\hline-0.333333 & 8.86839 & 43.0286 & $151.932 \pm 5.96048 \mathrm{E}-4$ & $437.509 \pm 4.88145 \mathrm{E}-3$ \\
\hline 0. & 7.33333 & 29.0435 & $82.998 \pm 7.61558 \mathrm{E}-4$ & $192.684 \pm 6.83182 \mathrm{E}-3$ \\
\hline
\end{tabular}

Table 4. Numerical values for the $\mathrm{O}\left(\epsilon^{-3}\right), \mathrm{O}\left(\epsilon^{-2}\right), \mathrm{O}\left(\epsilon^{-1}\right)$ and $\mathrm{O}\left(\epsilon^{0}\right)$ coefficients of $\mathcal{J}_{*} \mathcal{J}_{i k}(x ; \epsilon ; 1,3)$ for various values of $\log _{10}(Y)$. The numbers have been obtained from eq. (6.1), the semi-analytic one for the $\mathrm{O}\left(\epsilon^{-1}\right)$ coefficient and MB integrals for the $\mathrm{O}\left(\epsilon^{0}\right)$ coefficient. In the last two columns, we also show the numerical uncertainties as reported by MB.m. 
have shown are for demonstration purposes only and that obtaining the high resolution interpolating tables needed for the computation of an actual cross section is straightforward. Increasing the accuracy of each entry is feasible as well, if needed, thanks to the numerical integration options implemented in MB.m.

The integrals discussed in this paper appear when integrating the subtraction terms that regularize the real-virtual NNLO correction to the jet cross section, see ref. [18]. The final step in finishing the definition of the subtraction scheme is the computation of the integrated counterterms corresponding to the subtraction terms that regularize the doubly-real NNLO contribution, see ref. [19]. The iterated singly-unresolved counterterms (those labeled by $A_{12}$ in ref. [19]) are almost identical to the ones considered in this paper. As regards the doubly-unresolved counterterms (those labeled by $A_{2}$ in ref. [19]), some integrals that appear there are more cumbersome than the integrals presently considered, but nevertheless, their analytical structure is essentially the same. Therefore we expect that the techniques of the present paper will be straightforwardly applicable for the computation of these remaining contributions.

Files of our results can be obtained from the preprint server http://arXiv.org by downloading the source. They are also available at [41] or from the authors upon request.

\section{Acknowledgments}

We acknowledge useful discussions with J. Blümlein, T. Riemann and V. Yundin . This work is supported in part by the Deutsche Forschungsgemeinschaft in SFB/TR 9, the Helmholtz Gemeinschaft under contract VH-NG-105, the Hungarian Scientific Research Fund grand OTKA K-60432 and by the Swiss National Science Foundation (SNF) under contract 200020-117602.

\section{References}

[1] S. Catani and M.H. Seymour, A general algorithm for calculating jet cross sections in NLO QCD, Nucl. Phys. B 485 (1997) 291 [Erratum ibid. B 510 (1998) 503] [hep-ph/9605323] [SPIRES].

[2] A. Gehrmann-De Ridder, T. Gehrmann and E.W.N. Glover, Infrared structure of $e^{+} e^{-} \rightarrow 2$ jets at NNLO, Nucl. Phys. B 691 (2004) 195 [hep-ph/0403057] [SPIRES].

[3] S. Weinzierl, Subtraction terms at NNLO, JHEP 03 (2003) 062 [hep-ph/0302180] [SPIRES].

[4] S. Frixione and M. Grazzini, Subtraction at NNLO, JHEP 06 (2005) 010 [hep-ph/0411399] [SPIRES].

[5] G. Somogyi, Z. Trócsányi and V. Del Duca, Matching of singly- and doubly-unresolved limits of tree-level QCD squared matrix elements, JHEP 06 (2005) 024 [hep-ph/0502226] [SPIRES].

[6] A. Gehrmann-De Ridder, T. Gehrmann, E.W.N. Glover and G. Heinrich, Second-order QCD corrections to the thrust distribution, Phys. Rev. Lett. 99 (2007) 132002 [arXiv:0707.1285] [SPIRES]. 
[7] A. Gehrmann-De Ridder, T. Gehrmann, E.W.N. Glover and G. Heinrich, Jet rates in electron-positron annihilation at $\mathcal{O}\left(\alpha_{s}^{3}\right)$ in QCD, Phys. Rev. Lett. 100 (2008) 172001 [arXiv: 0802.0813] [SPIRES].

[8] S. Weinzierl, NNLO corrections to 3-jet observables in electron-positron annihilation, Phys. Rev. Lett. 101 (2008) 162001 [arXiv:0807.3241] [SPIRES].

[9] A. Gehrmann-De Ridder, T. Gehrmann, E.W.N. Glover and G. Heinrich, NNLO corrections to event shapes in $e^{+} e^{-}$annihilation, JHEP 12 (2007) 094 [arXiv:0711.4711] [SPIRES].

[10] S. Weinzierl, Event shapes and jet rates in electron-positron annihilation at NNLO, JHEP 06 (2009) 041 [arXiv: 0904.1077] [SPIRES].

[11] A. Gehrmann-De Ridder, T. Gehrmann and E.W.N. Glover, Gluon-gluon antenna functions from Higgs boson decay, Phys. Lett. B 612 (2005) 49 [hep-ph/0502110] [SPIRES].

[12] A. Gehrmann-De Ridder, T. Gehrmann and E.W.N. Glover, Quark-gluon antenna functions from neutralino decay, Phys. Lett. B 612 (2005) 36 [hep-ph/0501291] [SPIRES].

[13] A. Gehrmann-De Ridder, T. Gehrmann and E.W.N. Glover, Antenna subtraction at NNLO, JHEP 09 (2005) 056 [hep-ph/0505111] [SPIRES].

[14] S. Catani and M. Grazzini, An NNLO subtraction formalism in hadron collisions and its application to Higgs boson production at the LHC, Phys. Rev. Lett. 98 (2007) 222002 [hep-ph/0703012] [SPIRES].

[15] S. Catani, L. Cieri, G. Ferrera, D. de Florian and M. Grazzini, Vector boson production at hadron colliders: a fully exclusive QCD calculation at NNLO, arXiv:0903.2120 [SPIRES].

[16] S. Weinzierl, The infrared structure of $e^{+} e^{-} \rightarrow 3$ jets at NNLO reloaded, JHEP 07 (2009) 009 [arXiv:0904.1145] [SPIRES].

[17] G. Somogyi and Z. Trócsányi, A new subtraction scheme for computing QCD jet cross sections at next-to-leading order accuracy, hep-ph/0609041 [SPIRES].

[18] G. Somogyi and Z. Trócsányi, A subtraction scheme for computing QCD jet cross sections at NNLO: regularization of real-virtual emission, JHEP 01 (2007) 052 [hep-ph/0609043] [SPIRES].

[19] G. Somogyi, Z. Trócsányi and V. Del Duca, A subtraction scheme for computing QCD jet cross sections at NNLO: regularization of doubly-real emissions, JHEP 01 (2007) 070 [hep-ph/0609042] [SPIRES].

[20] G. Somogyi, Subtraction with hadronic initial states: an NNLO-compatible scheme, arXiv:0903.1218 [SPIRES].

[21] U. Aglietti, V. Del Duca, C. Duhr, G. Somogyi and Z. Trócsányi, Analytic integration of real-virtual counterterms in NNLO jet cross sections I, JHEP 09 (2008) 107 [arXiv:0807.0514] [SPIRES].

[22] G. Somogyi and Z. Trócsányi, A subtraction scheme for computing QCD jet cross sections at NNLO: integrating the subtraction terms I, JHEP 08 (2008) 042 [arXiv:0807.0509] [SPIRES].

[23] M.C. Bergere and Y.-M.P. Lam, Asymptotic expansion of Feynman amplitudes. Part 1: the convergent case, Commun. Math. Phys. 39 (1974) 1 [SPIRES].

[24] N.I. Usyukina, On a representation for three point function, Teor. Mat. Fiz. 22 (1975) 300 [SPIRES]. 
[25] E.E. Boos and A.I. Davydychev, A method of evaluating massive Feynman integrals, Theor. Math. Phys. 89 (1991) 1052 [Teor. Mat. Fiz. 89 (1991) 56] [SPIRES].

[26] V.A. Smirnov, Analytical result for dimensionally regularized massless on-shell double box, Phys. Lett. B 460 (1999) 397 [hep-ph/9905323] [SPIRES].

[27] J.B. Tausk, Non-planar massless two-loop Feynman diagrams with four on-shell legs, Phys. Lett. B 469 (1999) 225 [hep-ph/9909506] [SPIRES].

[28] V.A. Smirnov, Evaluating Feynman integrals, Springer Tracts Mod. Phys. 211 (2004) 1.

[29] M. Czakon, Automatized analytic continuation of Mellin-Barnes integrals, Comput. Phys. Commun. 175 (2006) 559 [hep-ph/0511200] [SPIRES].

[30] Z. Nagy, G. Somogyi and Z. Trócsányi, Separation of soft and collinear infrared limits of QCD squared matrix elements, hep-ph/0702273 [SPIRES].

[31] J. Gluza, K. Kajda and T. Riemann, AMBRE - a Mathematica package for the construction of Mellin-Barnes representations for Feynman integrals, Comput. Phys. Commun. 177 (2007) 879 [arXiv:0704.2423] [SPIRES].

[32] J.A.M. Vermaseren, Harmonic sums, Mellin transforms and integrals, Int. J. Mod. Phys. A 14 (1999) 2037 [hep-ph/9806280] [SPIRES].

[33] S. Moch, P. Uwer and S. Weinzierl, Nested sums, expansion of transcendental functions and multi-scale multi-loop integrals, J. Math. Phys. 43 (2002) 3363 [hep-ph/0110083] [SPIRES].

[34] S. Moch and P. Uwer, XSummer: transcendental functions and symbolic summation in Form, Comput. Phys. Commun. 174 (2006) 759 [math-ph/0508008] [SPIRES].

[35] E. Remiddi and J.A.M. Vermaseren, Harmonic polylogarithms, Int. J. Mod. Phys. A 15 (2000) 725 [hep-ph/9905237] [SPIRES].

[36] J. Gluza and T. Riemann, New results for 5-point functions, in the Proceedings of 2007 international Linear Collider Workshop (LCWSO7 and ILC07), Hamburg Germany May $30-$ June 3 2007, pg. LOOP01, arXiv:0712.2969 [SPIRES].

[37] J. Gluza and T. Riemann, A new treatment of mixed virtual and real IR-singularities, PoS (RAD COR 2007) 007 [arXiv:0801.4228] [SPIRES].

[38] L. Lewin, Polylogarithms and associated functions, North Holland, New York U.S.A. (1981) [ISBN: 0-444-00550-1].

[39] T. Hahn, CUBA: a library for multidimensional numerical integration, Comput. Phys. Commun. 168 (2005) 78 [hep-ph/0404043] [SPIRES].

[40] G. Heinrich, Sector decomposition, Int. J. Mod. Phys. A 23 (2008) 1457 [arXiv:0803.4177] [SPIRES] and references therein.

[41] DESY, webpage http://www-zeuthen.desy.de/theory/research/CAS.html. 